\title{
Multi-Bubble Universe Model: A Quantum-Relativistic Gravitational Theory of Space-Time
}

\author{
Massimo Auci1,2 \\ ${ }^{1}$ Maths and Physics Department, State European International School “Altiero Spinelli” Via Figlie dei Militari 25, Torino, Italy \\ ${ }^{2}$ Space Science Department, Odisseo-Space Via Battistotti Sassi 13, Milano, Italy \\ Email: massimo.auci@gmail.com
}

How to cite this paper: Auci, M. (2021) Multi-Bubble Universe Model: A Quantum-Relativistic Gravitational Theory of Space-Time. Journal of Modern Physics, 12, 179-217.

https://doi.org/10.4236/jmp.2021.123016

Received: October 4, 2020

Accepted: February 7, 2021

Published: February 10, 2021

Copyright $\odot 2021$ by author(s) and Scientific Research Publishing Inc. This work is licensed under the Creative Commons Attribution International License (CC BY 4.0).

http://creativecommons.org/licenses/by/4.0/

\begin{abstract}
A multi-bubble model of universe is presented, in which gravity is the result of the aging of electromagnetic dipoles produced in quantum fluctuations of the action inside a condensate of a particular type of balancing gravitons. In the model, the exchange of gravitons among the dipoles and the potential of vacuum balances the energy of the fluctuation. The effect is to create bubbles of space-time that by growing they expand the local vacuum. The model suggests that the universe originates from overlapping of bubbles of space-time associated with dipoles. Matter is originated during gravitons decay. The use of the Bridge Theory demonstrates how the attractive force field that emerges in each bubble is in the first approximation in accordance with Newtonian gravity at small, medium and large distances in accordance with the theory of General Relativity by also introducing a variable cosmological term that justifies some observed cosmological anomalies. The model overcomes the current concepts of Dark Energy and Dark Mass in favor of a gravity produced by the curvature of space-time of the bubble. The existence of the balancing gravitons provides an estimate of the actual amounts of Dark Energy, Dark Matter and matter measured in the current universe. The estimated theoretical mass of the balancing gravitons is consistent with the Kaluza-Klein gravitons of $2.68 \mathrm{TeV}$ observed in the ATLAS experiment during Run \#1. Moreover, the use of the observational data of the rotation speeds of two samples of galaxies allows to verify the good agreement of the real universe with the model, providing a possible explanation of the variability in the measurement of the Hubble constant.
\end{abstract}

\section{Keywords}

Bridge Theory, Cosmology, Dark Matter, Dark Energy, Gravitons, Relativity, Unification 


\section{Introduction}

In history of cosmology [1], the assumption of the Lambda-CDM (Cold Dark Matter) model as describing the physical reality of the universe, naturally leads to a first difficulty in understanding the behavior of gravity on a galactic, extragalactic and cosmic scale [2]. A distribution of a large quantity of Dark Matter is imagined to exist and be gravitationally active influencing the halo of each galaxy, manifesting its presence through an anomalous rotation speed of the galactic halo itself. A second difficulty is the problem of the origin of the universe. All instrumental observations made on the Cosmic Microwave Background (CMB) are consistent with an initial inflationary scenario in which expansion was initially very fast, after which the universe slowed down and accelerated again as if was driven by antigravity produced by a hypothesized Dark Energy, so in light of the observations of the WMAP and Plank spacecrafts and of the recent measurements of the Hubble constant performed by different methods, all inconsistent with those obtained on the CMB, to believe that the Lambda-CDM model is able to describe the universe at every scale correctly do not seem easy.

A consistency at the Lambda-CDM model is given by the observation of an amount of ordinary visible baryonic matter contributing with $\Omega_{B} \cong 0.05$ at the baryonic density, at which is necessary to add an amount of yet not identified Dark Matter contributing with a $\Omega_{D M} \cong 0.25$ to density and of an amount of Dark Energy, probably associated to the vacuum energy with a $\Omega_{D E} \cong 0.7$, both sufficient to produce a flat universe expanding with a velocity growing in time. These two characteristics involve two fundamental aspects of physics. The first is related to the not confirmed existence of neutral bosonic particles habiting the galactic halos with a Dark Matter role, the second of a vacuum, not exactly empty, that actively participates at the total energy of the universe causing its expansion.

For this, there are some deep difficulties for the acceptance of the Lambda-CDM model in the present form, in fact on a galactic scale and beyond, it is very difficult to reconcile the model predictions with the direct observations. It would seem that a set of cosmological constants cannot be sufficient to describe perfectly the behavior of the single galaxies, of the clusters and of the universe itself in its early stages. Despite this, the historical experimental confirmation from LIGO and VIRGO [3] collaborations of the existence of the gravitational waves, and the confirmation of the existence of a gravitational redshift along the orbit at pericenter of $\mathrm{S} 2$, when the star is nearest the intense gravity produced by the massive black-hole in Sgr A [4], they should eliminate any doubts about the physical reality and the goodness of the Theory of General Relativity. Thus, it is necessary to assume gravity not as a fundamental pseudo-Newtonian force, but as an emerging force produced by the local curvature of space-time induced by the presence of masses and energy in the vacuum as predicted by the field equation in which gravity appears as a non-quantized field. In this sense, gravity seems to be an exception on the scenario of the fundamental forces. In particular, 
if gravity is an effect and not a fundamental force, it is necessary to know why the curvature of space-time is produced and why it grows when the mass and density of energy grow in space-time, especially it is necessary to wonder why gravity is not a quantum field like other forces.

In this work, a new model of the universe is proposed and it is shown that the Theory of General Relativity reinterpreted in the context of a complete Bridge Theory (BT) [5] [6] is able to describe the universe without assuming the existence of real Dark Matter and real Dark Energy because their effects are emulated during the creation of space-time.

\section{Why Is It Necessary to Try to Use Another Approach to Describe a New Cosmological Model?}

One of the fundamental expected goals of modern physics is to discover gravity as a relativistic quantum force unified with other forces in nature. Two fundamental steps are needed to achieve this goal. The first is the experimental discovery of the existence of a quantum phenomenon associated with gravity or alternatively of a quantum theory of gravity describing the gravitational effects on each scale. The second is to understand as gravity can be unified with all the other forces, namely with the electromagnetic force. In both cases, to try to achieve these results it is necessary to start from a theory consistent with quantum theory, with relativity and with electromagnetic theory.

This work presents a model of universe in which electromagnetic forces and gravity are unified. The model developed with the use of quantum physics and relativity in the context of the BT is proved to be theoretically and phenomenologically consistent with the description of the electromagnetic quantum-relativistic phenomena as above hypothesized to be necessary. In any case, the use of BT based on real physical phenomena can be considered an interesting attempt to unify within a single non-speculative theory a quantum-gravitational phenomenology with electromagnetic forces.

\subsection{The Dipole as an Electromagnetic Model of the Photon Exchanged during a Particle-Antiparticle Electromagnetic Interaction}

The theoretical and phenomenological approach takes into account that in BT the electromagnetic interactions occur by means of the creation of real and virtual Dipole Electromagnetic Sources (DEMS), whose interaction energy is regulated by the wavelength defined by the minimum distance reached by the particles during their approach. The correctness of this approach proposed in terms of model in reference [7] and [8] and reviewed successively in Bridge Theory terms is guaranteed by the theoretical estimation of the Sommerfeld constant springing out by theory as a universal numerical constant independent by the values of the Planck constant, of the electron charge, of the light speed and by external adjustment of useful parameters. 
The DEMS model demonstrates the initial conjecture [9] based on the role of the transverse component of the Poynting vector associated with the induction zone during the direct interaction of the particle-antiparticle pair, in generating the quantization. In synthesis, the electromagnetic dipole formed during a direct interaction has a non-zero dipole moment that gives it cylindrical symmetry in the Poynting vector field. The lack of spherical symmetry with respect to radial emission gives rise to a non-null transverse component of the Poynting vector associated with a rotating wave that localizes energy and momentum equal to that defined for a photon within the first wavefront. More exactly, inside the source zone of the DEMS defined by a spherical crown limited by the radii $\lambda / 2<r<3 \lambda / 4$, an amount of energy formally and numerically in agreement with the photon $h c / \lambda$ is localized. The first value of the action was calculated in a manner completely consistent with the DEMS model. The action value $h_{1}=$ $6.626178 \times 10^{-34} \mathrm{~J}$.s derived from the theoretical value of the Sommerfeld's constant calculated by the model, was subsequently improved in the more wide context of the BT by introducing the idea that the interacting charges, if referred to the dipole, cannot be approximated to a geometric points, so considering their angular dispersion was possible to provide an improved value $h_{2}=6.626075 \times$ $10^{-34} \mathrm{~J}$.s of the action constant. This action value is in very good agreement with the current experimental value of the Planck constant $h_{\text {Planck }}=6.626071 \times 10^{-34}$ $\mathrm{J} \cdot \mathrm{s}$ demonstrating that the DEMS model is independent from external conditions and allows a theoretical definition of the $\alpha^{-1}$ Sommerfeld's constant as a computable fundamental constant in an universe free to evolve without constrains. In this way, the DEMS behaves as a quantum mediator of the energy exchanged in a direct electromagnetic interaction, by emulating the quantized exchange of energy and momentum between the interacting particles.

\subsection{The Role of the Uncertainty Principle in the Space-Time Creation}

Considering that in BT the phase of production of a DEMS, called alpha phase (A), localizes the energy and momentum of the photon exchanged by the interacting particles, using the Poynting theorem is proved the existence of an uncertainty principle for observers external to the first wavefront in accordance with the Heisenberg principle, but also of an uncertainty principle for internal observers imbedded in the electromagnetic field of the DEMS, in this case, the pairs of variables position-momentum and time-energy within the dipole behave as conjugate variables because each pair is associated with the Poynting vector field of the DEMS, characterizing the localization and the propagation of the electromagnetic field. Thus, the electromagnetic field that fills the bubble within the wavefront defines the space-time of the bubble itself.

When the phase A ends, the photon exchange ends too. During this phase the interacting particles exchanged an energy equal to that of a photon with wavelength $\lambda$, from this instant the pair of particles begin to move away from each 
other progressively increasing their mutual distance of interaction, i.e. they increase the wavelength of direct interaction up to infinity by bringing the DEMS up to zero energy. This final phase is called omega phase $(\Omega)$. It is necessary to ask ourselves how the energy of DEMS is stored and distributed in these two phases. In fact, during the alpha phase, the energy of interaction of the two particles is supplied externally by a process of acceleration, instead in the $\Omega$ phase, the first wavefront characterizing the DEMS energy emission is enclosing more and more space-time, while the wavelength that causally connects the two original interacting particles grows gradually reducing the electromagnetic energy of the actual DEMS. The difference in wavelength between the final and the initial signal defines the redshift of the original energy exchanged between the two interacting particles. If the energy conservation principle is not violated where did the missing energy go? In what other form of energy has the energy of the DEMS been transformed? This model suggests how the missing energy can become gravitational energy.

\section{Multi-Bubble Universe Model}

Considering the generalized uncertainty principle proposed in BT relative to the conjugate couples of variables energy-time and momentum-position measured by each observer, two independent cases can be considered:

1) The observer is not yet causally connected with the DEMS because it is moving externally to the first emitted wavefront of the DEMS in a pre-existing space-time, its position with respect to the DEMS is initially unknown. Reached by the wavefront the observer interacts with the DEMS through the wave. The interaction corresponds to an indirect measurement of the energy $E$ and momentum $P$ of the DEMS along the line of sight of the observer. In this case, the observations of momentum-position or of energy-time performed by each external observer, satisfy the Heisenberg's uncertainty principle. The relative observations of the position $r$ and time $t$ of both observers in order to the creation of the reciprocal DEMS, in agreement with BT are subject to the Lorentz-Einstein transformations. In this sense position and time of the observers depend on their relative movement with respect to the direct DEMS formation, the space-time in which each observer is imbedded is defined by the electromagnetic field produced by their interaction by means of the Planck action.

2) Two observers are placed in a pre-existing space-time and interacting with each other produce a new DEMS with respect to which they are placed inside the wave front emitted or equivalently, if a single observer is within the first wavefront of a DEMS the generalized uncertainty principle for internal observer in BT limits in both cases the measurable value of action of which the Planck constant is the upper limit, i.e. the direct interaction of a pair of particles or of a particle with one of the two particles of a DEMS, increasing the local action produces a weak perturbation of the pre-existing electromagnetic field rippling the space-time in terms of time-energy and position-momentum. The same phe- 
nomenology is also suitable to describe a pair of particles created in a fluctuation of action which through the DEMS produced, it describes the creation of a new space-time bubble enveloped by the wavefront of the source. In this sense, the current universe can be considered an overlap of single bubbles.

Assuming the early universe to be all the space-time created inside a primordial bubble crossed in time by a web of direct and indirect interactions formed by DEMS connecting each particle with an antiparticle-observer, the space-time can be though as an overlapping of bubbles. The Poynting's vector associated to the wavefront of each of these electromagnetic connections produces a directional pressure of radiation on the internal border of each local DEMS, with the effect to push on the resulting wavefront expanding the own space-time. Considering all the electromagnetic DEMS produced inside the universe, the resultant effect on the external border is a non-balanced pressure expanding it. When a direct connection produces a DEMS with wavelength $\lambda$, for the energy conservation principle, a part of the energy is involved in the DEMS production, if the DEMS is produced by a new pair of particles created from an action fluctuation, the amount of energy necessary for this creation must be taken somewhere, an energy supply that must exist before the emission of the pair and the formation of the DEMS, an energy made available from a field of nothingness, a sort of potential energy that feed the source zone of the DEMS emitting a wave increasing the space-time of the bubble. The external side of the wavefront corresponds to the border of a local space-time of the DEMS on which act simultaneously two antagonist pressures: one negative produced by the subtraction of energy from the nothingness field and one positive which produces the expansion of the wavefront. The two pressures are perfectly balanced in a way such that after the first acceleration during the production of the source zone of the DEMS, the velocity of expansion of the wavefront is steady and equal to the light speed in each direction respect each observer in agreement with the relativity principle.

The existence of a field of nothingness besides the field of electromagnetic vacuum is suggested by the fact that a pair of existing particles can interact in the vacuum, that is, in a pre-existing space-time, but it can also be created together with their space-time by an action fluctuation. In this case, the creation of the pair would increase the energy of the universe in addition to its extent, and this would violate the principle of conservation of the energy.

\subsection{Model of an Expanding Electromagnetic Space-Time Bubble}

Using BT and the DEMS model in which is proved as in the source zone of the dipole is localized an amount of energy and momentum equivalent to that of a photon of equal wavelength, let

$$
U=E+W
$$

to be the total amount of energy of vacuum inside a DEMS,

$$
E=h c / \lambda
$$


describes the electromagnetic energy localized in the photon exchanged between particle and antiparticle and $W$ the nothingness field that supplies the energy of the DEMS. In fact, for the conservation principle the electromagnetic energy (2) cannot be created but only transformed. After the creation the particles move apart each from other or annihilate in accordance with the conservation laws.

Following the theory, the creation of the pair is coinciding with a DEMS able of describing in BT the dual behavior of each particle [10]. The electromagnetic wave emitted by the source expands the electromagnetic bubble by pushing on the edge of the vacuum and inducing a constant radial resistance force $\sigma_{w}$ in all directions. The total strength in the solid angle is given by

$$
\frac{\mathrm{d} W}{\mathrm{~d} \lambda}=4 \pi \sigma_{w}
$$

which acts against the expansion of the wavefront such that the resultant radial strength of vacuum is null in each direction. This effect allows a wave propagation with a radial steady speed equal to light speed $c$. In fact, the wavefront grows up expanding with a force

$$
\frac{\mathrm{d} E}{\mathrm{~d} \lambda}=-\frac{h c}{\lambda^{2}}
$$

Considering the forces (3) and (4), the total strength

$$
\frac{\mathrm{d} U}{\mathrm{~d} \lambda}=-\frac{h c}{\lambda^{2}}+4 \pi \sigma_{w}
$$

is nulled for the wavelength $\lambda_{m}$ characterizing the wavelength of the minimum of energy of the vacuum corresponding to the ground state $U_{0}$ of the energy (1), for each other value of wavelength the strength (5) is different by zero and the total vacuum energy is greater than the ground state. Considering a stretching of the wavelength, integrating the strength (5) between an initial and a final wavelength $\left[\lambda, \lambda^{\prime}\right]$, to conserve unchanged the energy of the ground state must be

$$
\delta U=\left(\frac{h c}{\lambda^{\prime}}+4 \pi \sigma_{w} \lambda^{\prime}\right)-\left(\frac{h c}{\lambda}+4 \pi \sigma_{w} \lambda\right)=0
$$

In agreement with the Equations (1), (2) and (3)

$$
U=\frac{h c}{\lambda}+4 \pi \sigma_{w} \lambda
$$

is the total vacuum energy inside the bubble of space-time of a DEMS.

In order to maintain the energy of the state (7) unchanged during the wavelength stretching, it is necessary that the transition occurs adiabatically. Assuming the state $U$ as a constant value, the Equation (7) gives as solutions two wavelength corresponding to an initial and a final value of wavelength:

$$
\lambda_{i, f}=\frac{U \pm \sqrt{U^{2}-16 \pi \sigma_{w} h c}}{8 \pi \sigma_{w}}
$$

only when $U$ is such a that

$$
U \geq \sqrt{16 \pi \sigma_{w} h c}
$$


According to the Equation (9), considering an action fluctuation of arbitrary value $H$, is possible to define the energy of the vacuum as

$$
U=2 \sqrt{4 \pi \sigma_{w}(h+H) c}
$$

the wavelengths (8) become

$$
\lambda_{i, f}=\sqrt{\frac{(h+H) c}{4 \pi \sigma_{w}}} \mp \sqrt{\frac{H c}{4 \pi \sigma_{w}}}
$$

For $H=0$ (no fluctuation), the Equation (11) gives $\lambda_{m}=\lambda_{i}=\lambda_{f}$, both the wavelengths are coinciding and are corresponding with the ground state of energy

$$
U_{0}=2 \sqrt{4 \pi \sigma_{w} h c}
$$

with

$$
\lambda_{m}=\sqrt{\frac{h c}{4 \pi \sigma_{w}}} .
$$

In this sense, electromagnetic energy (2) possibly emitted in a fluctuation through a local DEMS, must be supplied by an amount of energy of the nothingness field by means of an increase of action $H>0$, i.e. the energy of the electromagnetic vacuum grows and it is $E>W$, therefore the wavelength of the DEMS would become lower than its limit value $\lambda_{m}$, but a quantity of energy is returned to the nothingness field inducing an adiabatic transition that reduces the DEMS energy stretching the wavelength from the initial value $\lambda_{i}$ to the final value $\lambda_{f}$ decreasing the electromagnetic energy and increasing the energy of the nothingness field in such a way that $E<W$. Using the Equation (7), (11) and (13), it is possible to verify the identity of the vacuum energy first and after the transition $U_{i}=U_{f}$ for each arbitrary fluctuation $\mathrm{H}$ proving the total energy (7) grows but the transition leaves it unchanged

$$
U_{i, f}=2 \sqrt{4 \pi \sigma_{w}(h+H) c}
$$

Assuming fluctuations $h+H$ with values multiple of the Planck action constant, defining arbitrarily

$$
H=(n-1) h
$$

with $n \geq 1$, using Equation (11) only spontaneous transitions $\lambda_{i} \rightarrow \lambda_{f}$ occurring from an initial value

$$
\lambda_{i}=(\sqrt{n}-\sqrt{n-1}) \lambda_{m}
$$

towards a final value

$$
\lambda_{f}=(\sqrt{n}+\sqrt{n-1}) \lambda_{m}
$$

with a finite but not arbitrary increment of wavelength

$$
\delta \lambda=2 \sqrt{n-1} \lambda_{m}
$$

produced by adiabatic transitions able to maintain unchanged during the transition the in initial state of the vacuum energy (7). Using the Equations (14), (15) 
and Equation (16) the electromagnetic and nothingness energies of the local vacuum become respectively

$$
\begin{aligned}
E_{i, f} & =\frac{1}{2} U_{0}(\sqrt{n} \pm \sqrt{n-1}) \\
W_{i, f} & =\frac{1}{2} U_{0}(\sqrt{n} \mp \sqrt{n-1})
\end{aligned}
$$

yielding for the total energy of vacuum (14)

$$
U_{i, f}=U_{0} \sqrt{n}
$$

which is a function of the multiplicity $n \geq 1$. In each case, in agreement with Equation (6) the transition occurs adiabatically

$$
\delta U=U_{f}-U_{i}=0
$$

implying during the transition using the Equation (18) and (19) and (21)

$$
-\delta E=\delta W=U_{0} \sqrt{n-1}
$$

i.e. each increase of wavelength (17) produces a decrease of the electromagnetic energy of the DEMS balanced by the increase of the energy of the nothingness field. The number $n$ defines the energy of the fluctuation and the vacuum state (20). In the ground state $n=1$ the electromagnetic and nothingness energies are identical, each transition occurring not modifies the final wavelength of the DEMS which remains stable at the minimum energy, for this characteristic the quantum of energy that can be exchanged between the electromagnetic fields of the vacuum and the nothingness field is defined Balancing Graviton (BG) and it is associate to a boson particle $G_{B G}$ with energy

$$
E_{B G}=\sqrt{4 \pi \sigma_{w} h c} .
$$

Using the Equation (23), the Equation (12) becomes

$$
U_{0}=2 E_{B G} \text {. }
$$

Vice versa, for fluctuations with $n>1$, the electromagnetic energy (18) is greater than the nothingness energy (19) and a number of BG (23) are exchanged increasing the energy of the nothingness field, expanding space-time and maintaining unchanged the initial energy of the vacuum state after the fluctuation. Now defining usefully $n=N^{2}+1$ the integer number $N=0,1,2,3, \cdots$ is the number of BG exchanged between the electromagnetic field of the DEMS and the nothingness field. After the transition the final energy of the vacuum state is:

$$
U=U_{0} \sqrt{N^{2}+1} .
$$

Using the definition of the number of BG exchanged the Equation (22) becomes

$$
-\delta E=\delta W=N U_{0} .
$$

Higher is the initial electromagnetic energy (18) with multiple fluctuations $n=2,5,10, \cdots$, greater is the number $N=\sqrt{n-1}$ of BG that are exchanged 
during the adiabatic transition between the DEMS and the nothingness field, lower is the residual electromagnetic energy after the transition, in which the wavelength of the original DEMS is stretched of a length increase defined by the Equation (17) with a redshift

$$
z=2 N\left(\sqrt{N^{2}+1}+N\right) .
$$

In other terms, by the exchange of $N \geq 1$ BG, the bubble with radius coinciding with the initial wavelength $\lambda_{i}$ of the DEMS has a quantized expansion with an increase of radius up to $\lambda_{f}$ and an increase of space-time, converting the part in excess of electromagnetic energy of the DEMS in energy of the nothingness field which corresponds to a growing tension on the internal border of the space-time. In this model the nothingness energy has the role of potential energy of the vacuum.

\subsection{Balancing Gravitons and Action Fluctuations}

In order to evaluate the constant strength $\sigma_{w}$ is necessary to assume that with a very high level of electromagnetic energy density within an expanding primary spherical bubble, the nothingness field can have a number of BG with an energy enough high to produce new fluctuations forming each a new DEMS transforming the original space-time inside the primary bubble in an overlapping of sources equivalent to a gas of photons, an overlap of bubbles ping in which each photon has a wavelength less equal than that of the primary bubble. This condition originates a black-body spectrum with a characteristic cut-off wavelength as defined in BT. The Planck spectrum is characterized by the average temperature $T_{\text {ave }}=\frac{h c}{k_{B} \lambda_{\text {ave }}}$ with electromagnetic emissions within two cutting frequencies, a lower frequency due to the upper wavelength of the bubble and an upper frequency beyond which the DEMS becomes a Micro-Black-Holes (MBH) [11]. Since is not correct to use directly the results obtained for a $\mathrm{MBH}$ in which gravity has been assumed as a Newtonian fundamental strength, it is possible to estimate the cut-off frequency i.e. the minimum value of wavelength, considering when the total electromagnetic energy localized by the DEMS becomes equivalent to all the energy carried by the impinging interacting particle and simultaneously equal to the Newtonian gravitational energy of the interacting pair of particles emerging by the interaction. To solve these three conditions:

$$
\left\{\begin{array}{l}
E=\frac{h c}{\lambda} \\
E=m c^{2} \\
E=G_{0} \frac{m^{2}}{\lambda}
\end{array}\right.
$$

where $G_{0}$ is an initial unknown value of the gravitational coupling constant acting inside the primary DEMS of space-time or in other terms, the early value of the gravitational constant in a primary bubble. Using the three previous equa- 
tions in pairs of two, the Equation (28) yields a pseudo-Planck mass

$$
m_{e q}=\sqrt{\frac{h c}{G_{0}}}
$$

associated to the pseudo-Planck length defining the BG wavelength

$$
\lambda_{B G}=\sqrt{\frac{h G_{0}}{c^{3}}}
$$

Let the wavelength (13) $\lambda_{m} \equiv \lambda_{B G}$ to be the minimum non-zero wavelength associated at the minimum value of the nothingness energy achieving in space-time, the Equation (13) and (30) yield a constant force as the value of the total tension of space-time border when the bubble is forced to expanding

$$
\frac{\mathrm{d} W}{\mathrm{~d} \lambda}=\frac{c^{4}}{G_{0}}
$$

Consequently, in the ground state the energy of the nothingness field and of the electromagnetic field of vacuum state are equal to the energy (23) of a BG, i.e.

$$
E_{B G}=E_{0} \equiv W_{0}=\sqrt{\frac{h c^{5}}{G_{0}}}
$$

Equation (32) is the energy of a collapsed DEMS not coinciding numerically with that one defined for a $\mathrm{MBH}$ because the effective value of $G_{0}$ to use in the Equation (32) is yet to evaluate.

Since the energy of the BG (32) is the largest amount of electromagnetic energy that can be produced and localized within a DEMS, but it is also the lower energy achievable for the nothingness field, a fluctuation of action $H>0$ emerges under form of a one or more DEMS with high energy that cannot overcoming the energy limit of a BG, then emits the excess of energy in the form of an integer number of BG, the energy is absorbed from the field of nothing by stabilizing the electromagnetic energy of the DEMS, stretching the wavelength of the primary DEMS and expanding the bubble. After the fluctuation, the space-time is grown maintaining unchanged the initial total energy of vacuum. On the other hand, the nothingness field acquiring the energy of a great number of BG can re-provide energy to the electromagnetic field within the bubble, producing pairs of particles originating new DEMS and space-time inside the primary bubble. The process in its whole is a sort of energy recycling starting from the initial Zero Point Energy (ZPE) of the electromagnetic vacuum. For this way to behave, the BG exchange divides the energy between the electromagnetic field and the field of nothingness, keeping the total energy of the vacuum in constant balance. For the previous reason, the BG is here defined as a quantum of balance.

In the BG exchange, considering an adiabatic expansion in a space-time with $n \gg 1$, using the Equation (26), let

$$
\delta E=-N U_{0}
$$

and 


$$
\delta W=N U_{0}
$$

to be respectively the electromagnetic energy subtracted and the energy acquired by the nothingness field under form of gravitational potential energy by means of the exchange of BG, using the Equation (33) and (34) the Equation (21) is verified ever null for each adiabatic transition occurring by the exchange of an arbitrary numbers $N$ of $\mathrm{BG}$, i.e. the transition occurring during the fluctuation leaves the final energy unchanged, but the number $N$ of BG that define the total energy of the vacuum exchanged grows with the final effect of to stretch the wavelengths of the black-body spectrum as estimated with the (27).

\section{Local Pseudo-Gravity}

Considering a DEMS with an internal electromagnetic energy $E \ll E_{B G}$ and the Equation (7) in the form

$$
U=E+\frac{c^{4}}{G_{0}} \lambda
$$

where lower is the electromagnetic energy $E$, higher is the nothingness energy $W$, and as shown in Figure 1 the total energy of vacuum $U$ at right side of the events horizon grows expanding the wavelength of the corresponding DEMS. In the Eq. (35) the strength $c^{4} / G_{0}$ turn out to be a tension constant of the space-time fabric [12]. Considering only low energy events occurring inside a pre-existing space-time with a bubble of radius $\lambda$ associated with the emission of pairs of particles, of pairs of gamma or at radio waves, the wavelength characterizing the event is stretched by the expansion of the bubble in which it is contained without the exchanging of BG with the nothingness field. In this case, the energetical degrade of the bubble can be considered the elapsed time from the origin of the bubble. Time in this context can be considered an emergent energetic fourth dimension produced by the variation of the local electromagnetic energy with the stretching of the wavelength of the bubble and the increasing of the total tension of space-time.

Considering a bubble stretched from $\lambda$ to $\lambda$ ' with a continue redshift $z$, using the Equation (35) is possible to evaluate the electromagnetic energy measured by an observer along its sight line after the stretching of the bubble. Considering an electromagnetic energy variations, since $\delta U=0$ it follows $-\delta E=\delta W$ calculated between two different wavelengths $\lambda^{\prime}>\lambda$ with $-\delta E<E_{B G}$, it follows:

$$
E-E^{\prime}=\frac{c^{4}}{G_{0}} \lambda^{\prime}-\frac{c^{4}}{G_{0}} \lambda
$$

that yields

$$
E^{\prime}=E-z \frac{c^{4}}{G_{0}} \lambda
$$

corresponding to the redshifted observed energy estimated starting from the initial value $E$ reduced of an amount of energy proportional to the stretching work of the wavelength of the bubble. 


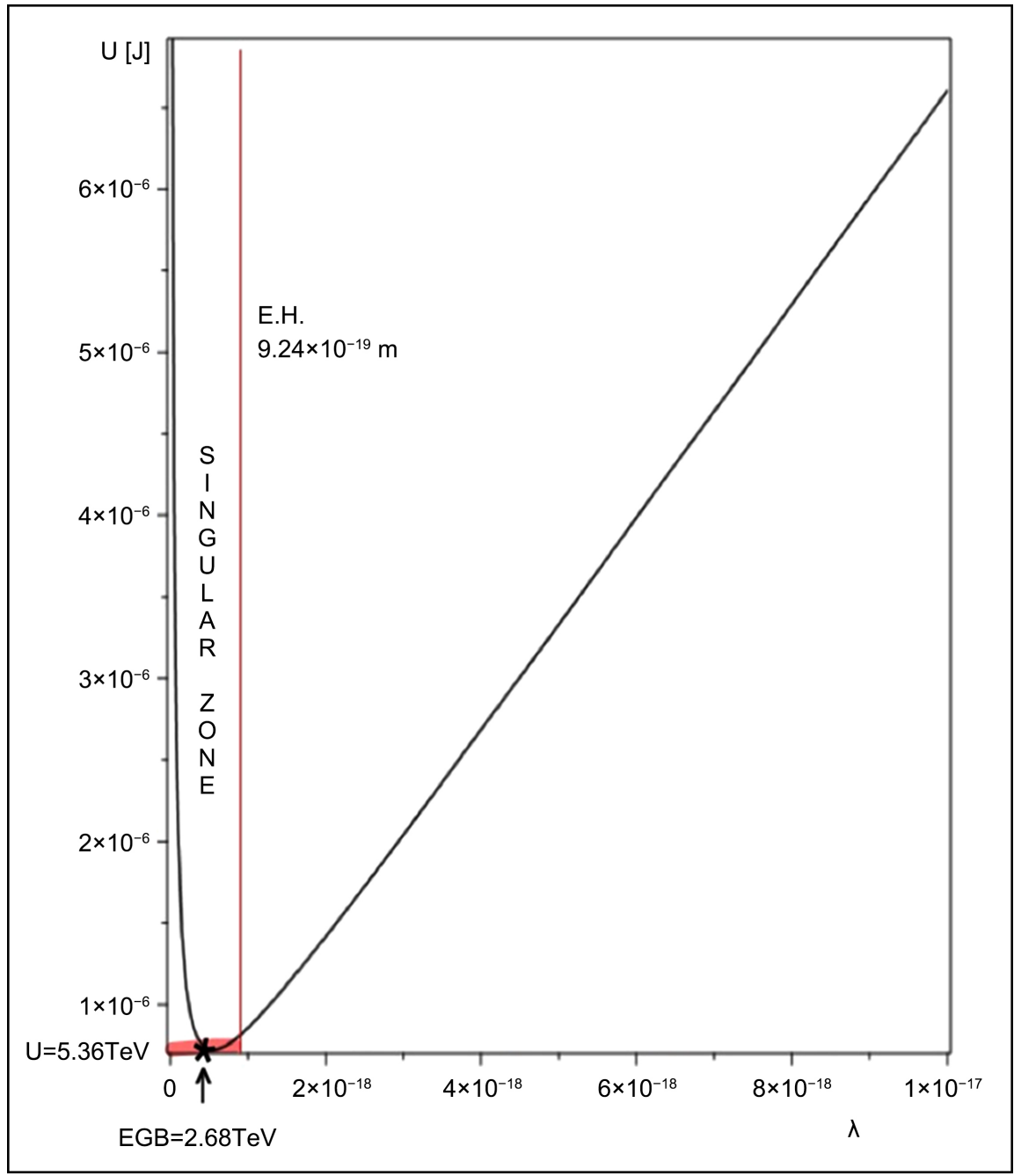

Figure 1. The total energy of the vacuum $U$ as a function of the wavelength of the DEMS. The figure highlights the singular zone under the events horizon and the BG energy (32) as estimated below.

Considering each electromagnetic indirect or direct connection between moving observers obtained throughout a causal electromagnetic interaction described relativistically with the use of the Doppler equation [6]:

$$
E_{\Gamma}=\frac{h c}{\lambda_{0}}=\frac{E^{2}-P^{2} c^{2}}{2(E-P c \cos \theta)}
$$

where $\lambda_{0}$ is the generalized Compton wavelength of a photon exchanged inside the original DEMS along the sight line connecting one component of the DEMS and the observer. Combining the redshifted energy (37) and the observed energy (38), the energy of the photon $E_{\Gamma}$ can be evaluated using the value of the original energy as proposed below.

\subsection{Pseudo-Gravity Field}

To describe the gravity phenomenology, it is necessary to consider an observer 
like in the case (1) of paragraph 3, that is an observer placed externally to a DEMS in a pre-existent space-time. When the observer is reached by the electromagnetic wave of the DEMS, an indirect connection between DEMS and observer is realized. The effect is a causal connection of the observer with one of the two particles forming the primary DEMS, this new connection is a secondary DEMS imbedded in the pre-existing space-time involving the primary source and its observer. An exchange of a photon of energy (38) occurs. After the eventual alpha phase, the omega phase of the DEMS starts at $\theta=\frac{\pi}{2}$ and the interaction angle is every $\theta>\frac{\pi}{2}$, i.e. the interaction angle between the trajectories of motion of the two interacting components of the secondary DEMS can be settled as $\theta=\pi / 2+\varphi$ where $\varphi$ it is a positive angle with respect to the radial line of sight, with which the observer moves away.

Using the definitions of total rest energy and of Compton energy given in Ref. [6], the numerator of the Equation (38) becomes

$$
E^{2}-P^{2} c^{2} \equiv\left(\frac{2 h c}{\lambda_{c}}\right)^{2}=\varepsilon^{2}
$$

which is the square of the rest energy of the interacting particles of the DEMS.

To describe a redshifted energy received along the sight line of the observer, is necessary to use the Equation (39) in which the energy characterizes the radius of the bubble. The rest energy (39) it is an invariant, so the Equation (38) for the received energy, becomes:

$$
E_{\Gamma}=\frac{\varepsilon^{2}}{2\left(\frac{h c}{\lambda}-z \frac{c^{4}}{G_{0}} \lambda\right)(1+\beta \sin \varphi)}
$$

Considering the electromagnetic vacuum within a bubble to be an initial space-time at temperature $T$, the successive casual production of new DEMS inside the primary bubble behaves as a gas of photons, that they will make it evolve up to become a black-body at the same mean temperature with a Planck spectrum associated to an average wavelength $\lambda_{\text {ave }}=h c / k_{B} T$.

The denominator of the Equation (40) allows to define as a function of the redshift $z$ of the light received, the border which divides the region of gravity from that of antigravity. So, using the mean characteristic wavelength and the definitions (30) and (32), the ratio

$$
\zeta_{B G}=\lambda_{B G}^{2} / \lambda_{\text {ave }}^{2} \equiv k_{B}^{2} T^{2} / E_{B G}^{2}
$$

represents a variable threshold that separates antigravitational from gravitational energy of the primary DEMS. In fact, using the Equation (41), the Equation (40) can be broken into three equations, each for a different zone of redshift in the same bubble at temperature $T$ : 


$$
E_{\Gamma}=\left\{\begin{array}{lc}
\frac{h c}{\lambda_{c}} & z=0 \\
\frac{G_{0}}{4 c^{4} \frac{\varepsilon^{2}}{\frac{1}{2}\left(\zeta_{B G}-z\right)(1+\beta \sin \varphi) \lambda}>0} & 0<z<\zeta_{B G} \\
\frac{G_{0}}{4 c^{4} \frac{1}{\frac{1}{2}\left(z-\zeta_{B G}\right)(1+\beta \sin \varphi) \lambda}<0} & z>\zeta_{B G}
\end{array}\right.
$$

Considering the space-time of a primary bubble, for each DEMS produced that emits inside, an observer can be placed within:

1) Quantum zone: at redshift $z=0$ the particles with resting energy (39) are not relativistic, the observer is one of the two interacting particles inside the DEMS or it is imbedded in their space-time. The energy is described by the Equation (42-a) and is equivalent to the energy of a photon with Compton wavelength, exchanged between the observer and one of the two interacting particles;

2) Anti-gravitational zone: with redshift $0<z<\zeta_{B G}$, the energy is positive and it is described by the Equation (42-b). The bubble is in very fast expansion. The observer and the particles acquire enough energy to escape, radially stretching the wavelength with which they interact;

3) Gravitational zone: with redshift $z>\zeta_{B G}$, the energy is negative and it is described by the Equation (42-c). Gravity is produced.

From the Equation (42-b) is evident that each bubble suffers a rapid expansion during the DEMS formation until the redshift does not satisfy the condition $\zeta_{B G}-z<0$ as required in the Equation (42-c). Considering the Equation (41) and the definition of redshift, the previous inequality has solution $\lambda>1.4656 \lambda_{B G}$ corresponds to $z>\zeta_{B G} \cong 0.4656$. When that occurs, antigravity becomes gravity. Considering the Events Horizon (EH) of a $\mathrm{BG}$ with a radius equal to the Schwarzschild radius $r_{E H}=2 \lambda_{B G}$, for wavelength in the interval $1.4656 \lambda_{B G}<\lambda \leq$ $2 \lambda_{B G}$ the DEMS produced are every below their EH and only by wavelength $\lambda>2 \lambda_{B G}$ the energy of the electromagnetic field emitted can propagate as shown in Figure 1.

\subsection{Effective Interaction Distance}

Considering the Equation (42-c), let $\lambda$ to be the minimum distance at which two particles start to interact and $\lambda^{\prime}$ the actual final distance achieved when the two particles move apart each to the other with speed $\beta$ and angle $\varphi$, is possible to define the effective interaction distance as the radial distance

$$
r=\frac{1}{2}\left(z-\zeta_{B G}\right)(1+\beta \sin \varphi) \lambda
$$

which in the zone (3) for relativistic particles with $\varphi=\pi / 2$, Equation (43) can be correctly approximated at $r \cong z \lambda$ and for non-relativistic particles at $r \cong z \lambda / 2$ proving that two observers indirectly connected in zone (3) are placed at an effective interaction distance of the order of the difference between the 
stretched final wavelength and the initial one. Considering an elapsed time from the end of the phase alpha, such that $\lambda^{\prime} \gg \lambda$, the effective distance (43) is of the order of the final wavelength $r \approx \lambda^{\prime}$, so the Equation (43) allows to describe the gravitational interaction energy $(42-c)$ in terms of the actual final distance measured as a function of four parameters $\left(z, \zeta_{B G}, \beta, \varphi\right)$.

Using the definitions of entangled rest masses (A.2) given in Appendix A and using the distance (43), the energy (42-c) can be rewritten in the pseudo-Newtonian form

$$
E_{\Gamma}=-G_{0} \frac{\mu_{1} \mu_{2}}{r}
$$

Defining the gravitational potential as $\phi_{i}=E_{\Gamma} / \mu_{j}$, the evaluation of the gravitational field yields

$$
\boldsymbol{g}_{1}=-\operatorname{grad} \phi_{1}=-G_{0} \frac{\mu_{1}}{r^{2}} \hat{\boldsymbol{r}}
$$

and reciprocally, for the body \#1

$$
\boldsymbol{g}_{2}=-\operatorname{grad} \phi_{2}=-G_{0} \frac{\mu_{2}}{r^{2}} \hat{\boldsymbol{r}}
$$

hence the mutual strength between two entangled particles of masses $\mu_{i}$ and $\mu_{j}$ placed at the effective distance (43) agrees with the Newtonian form of the universal gravitational law:

$$
\boldsymbol{F}=-G_{0} \frac{\mu_{i} \mu_{j}}{r^{2}} \hat{\boldsymbol{r}}
$$

The Equation (47) becomes a singularity at the border when the effective interaction distance is zero at $z=\zeta_{B G}$ independently from the value of the black-body temperature. Using Equation (47) and Equation (43) it is easy to prove that identical pairs of interacting particles producing identical gravitational attractive force are not necessarily interacting at a same final wavelength. In fact, their effective interaction distances do not depend on the only real space position, because identical values of gravitational force can be produced by an infinity of different combinations of values of the four parameters and of the wavelength from which they depend. This can assign equal values of energy and of gravity strength at different pairs of particles also if they are placed at a different real reciprocal distance in space-time. It is important to highlight as Equation (47) describes the gravitational force between two interacting particles as if their actual distance were fixed. Considering the original DEMS, when the two interacting particles are in relative motion it is necessary to consider that the value of the wavelength corresponding to the initial direct interaction has not a fixed value, but changes continuously as a function of their characteristic redshift. This changing occurs also for indirect interactions of observers connected through a DEMS by means of the propagation of the emitted electromagnetic wave. In this case, when the observer is achieved by the wave becomes connected at a DEMS and a direct interaction starts. In both cases it is necessary to correct 
the strength for the changing of scale of the interaction.

\subsection{Difference between Indirect and Direct Gravitational Interaction}

Considering an observer in the case (1) of paragraph 3 placed in a position in space-time much far from a DEMS, its distance can be expressed as a multiple of the effective wavelength at which the DEMS emits a wave respect the observer. Assuming the travelled distance

$$
r^{\prime}=\xi r
$$

where $r$ is the effective wavelength (43) of the gravitational wave signal emitted by the DEMS and $\xi>1$ a dimensionless fraction. When the wave signal reaches the observer a secondary DEMS with wavelength (48) starts to emits. The energy directly exchanged is of types (42-a). Successively, considering the relative motion of the two entangled partners, the interaction can be described by Equation (42-b) in which the bubble expands stretching its wavelength. When the condition $z>\zeta_{B G}$ is satisfied, the observer suffers an attractive force consistent with (47). In this case, the measured strength is attenuated than a factor $1 / \xi^{2}$ compared to that of the primary DEMS

$$
\boldsymbol{\Phi}=-G_{0} \frac{\mu_{i} \mu_{j}}{r^{\prime 2}} \hat{\boldsymbol{r}}=\frac{1}{\xi^{2}} \boldsymbol{F}
$$

Using the Equation (49), it is easy to verify that considering identical interacting particles distributed in space-time, when the energy is shared by wave, each interaction switches from indirect to direct and the energy propagated is reduced of a factor $1 / \xi$ per each interaction, while the gravitational force is reduced in strength of a factor $1 / \xi^{2}$ at a time producing entanglement among all the particles involved. Considering a different multiplicity for each interaction, the signal intensity is attenuated by the creation of secondary DEMS that absorb and degrade energy more quickly than happens for propagated waves that do not meet matter.

\subsection{Dynamic Correction of the Gravitational Strength}

When a direct gravitational interaction (42-c) between two particles starts, their relative motion in space-time stretches the original wavelength settled by their initial causal contact modifying continuously the gravitational strength acting between the two particles. To describe the changing in scale of the force occurring at charge of the work done to stretch the initial wavelength, it is necessary to consider the variation of the energy (44) respect the wavelength, i.e. respect the distance of the initial direct interaction. As shown in Appendix B, the resultant effective gravitational force is given by:

$$
\Psi=\frac{1}{2}\left(z+\zeta_{B G}\right)(1+\beta \sin \varphi) \boldsymbol{F}
$$

where the Equation (50) describes the correct radial force along the sight line of 
the observer placed at an effective distance $r$ from the particle with which it is entangled. Using the scalar dimensionless ratio

$$
Z=\frac{\Psi}{F}
$$

is possible to define the effective gravitational strength between two bodies in relative motion as represented in Figure 2. In other words, two particles placed at a direct interaction distance $r$ as defined in Equation (43), are attracted each to the other with an effective strength

$$
\Psi=-G_{0} Z \frac{\mu_{1} \mu_{2}}{r^{2}}
$$

Equation (52) is homolog to the Newton's law but it is different in respect the Equation (47) by the presence of the ratio (51) that can be assumed generally different. Attributing the changing in scale at the value of the gravitational pseudo-constant $G=G_{0} Z$, the Equation (52) allows to define the gravitational coupling pseudo-constant that varies in space-time with the change of parameters $\left(z, \zeta_{B G}, \beta, \phi\right)$. Using the Equations (51) and (52), let $b=\beta \sin \varphi$ with $0<$ $b<1$ the speed projection, the value of the gravitational pseudo-constant is defined by the function

$$
G=\frac{G_{0}}{2}\left(z+\zeta_{B G}\right)(1+b)
$$

Considering the local universe as represented in Figure 2(b), respect an observer in a lab at the present time, the gravitational coupling constant (53) must coincide at the actual (starred ${ }^{\star}$ ) standard value, i.e. $G^{\star}=6.67192 \times 10^{-11} \mathrm{~N} \cdot \mathrm{m}^{2} \cdot \mathrm{kg}^{-2}$ [13]. Considering the set of reduced parameters $\left(z \geq \zeta_{B G}, \zeta_{B G}, 0\right)$, using the Equation (32) and (41) with an actual black body temperature $T=2.73 \mathrm{~K}$ of the

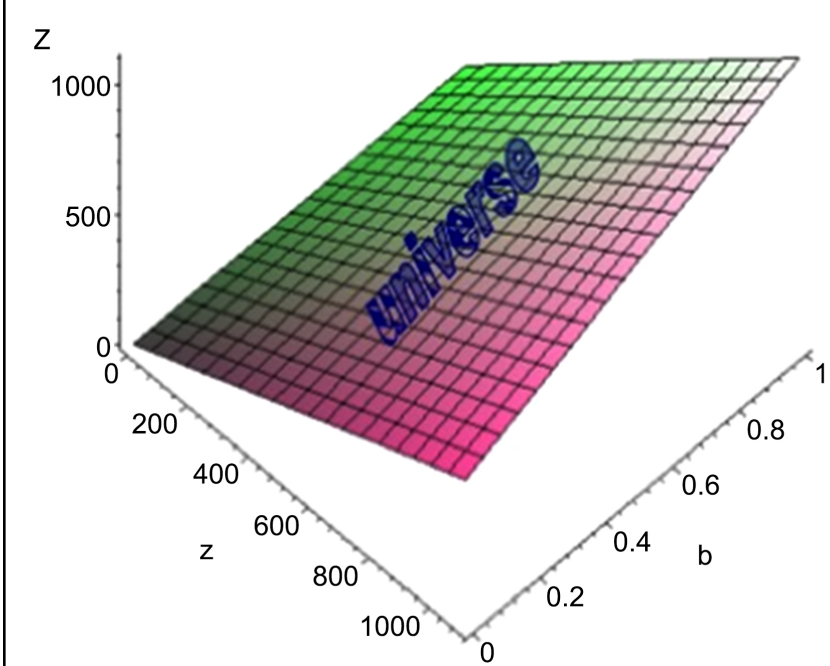

(a)

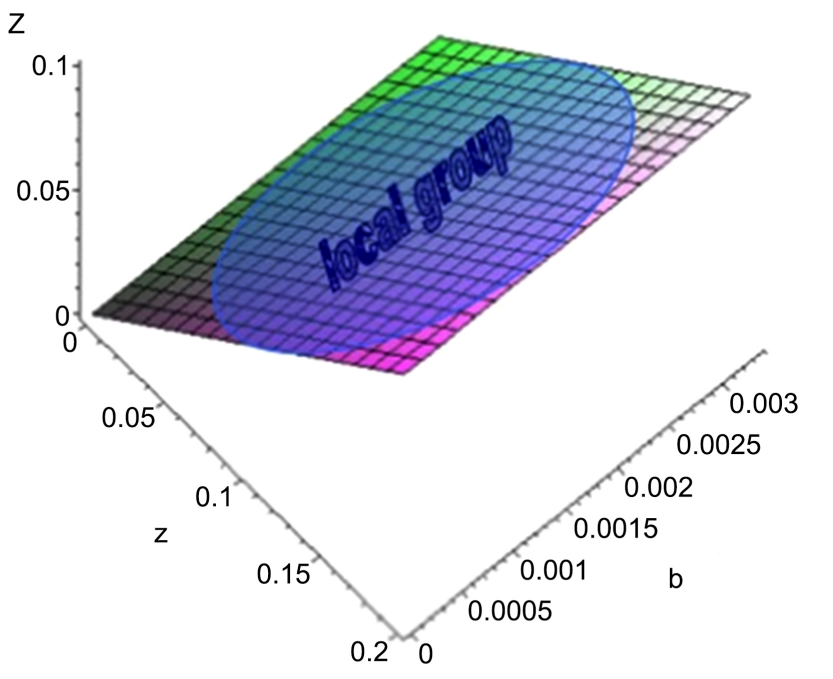

(b)

Figure 2. Representation of the variability of the $Z$ scaling factor modulating the early gravitational coupling constant $G_{0}$. (a) Scaling range for the whole universe. (b) scaling range for the local group. 
$\mathrm{CMB}$, the primordial gravitational coupling constant results to be $G_{0}=8.68 \times$ $10^{+21} \mathrm{~N} \cdot \mathrm{m}^{2} \cdot \mathrm{kg}^{-2}$ with $z \geq \zeta_{B G}=7.69 \times 10^{-33}$, from which, the nowadays value of the gravitational constant results to be as the expected actual one $G \geq G_{0} \zeta_{B G}=$ $6.67 \times 10^{-11} \mathrm{~N} \cdot \mathrm{m}^{2} \cdot \mathrm{kg}^{-2}$. Using the Equation (47), the local effective gravitational tension (50) between two interacting bodies can be rewritten in the Newtonian scalar form as

$$
\Psi_{i, j}=-G \frac{\mu_{i} \mu_{j}}{r^{2}}
$$

Considering as general fundamental cases, when the local interaction is relativistic $\left(\zeta_{B G}, \zeta_{B G}, 1\right)$ and when the local interaction is classic $\left(\zeta_{B G}, \zeta_{B G}, 0\right)$, Equation (54) yields in the first case

$$
\Psi \cong-2 G \frac{\mu_{i} \mu_{j}}{r^{2}} \hat{\boldsymbol{r}}
$$

and in the last

$$
\Psi \cong-G \frac{\mu_{i} \mu_{j}}{r^{2}} \hat{\boldsymbol{r}}
$$

but are possible many variations, for example for a captured electron orbiting around a proton with $\varphi \cong 0$, it follows $b=0$, assuming a vacuum temperature of $2.73 \mathrm{~K}$ with $z \cong \beta=\frac{1}{137} \gg \zeta_{G B}$ as suggested in reference [14], the Equation (53) gives a gravitational constant $G \cong G_{0} / 274=3.17 \times 10^{+19} \mathrm{~N} \cdot \mathrm{m}^{2} \cdot \mathrm{kg}^{-2}$ giving for the entangled masses $\mu_{e} \mu_{p}$ of a couple electron-proton in a hydrogen, a strength

$$
\Psi \cong-G \frac{\mu_{e} \mu_{p}}{r_{H}^{2}} \cong 1.73 \times 10^{-17} \mathrm{~N}
$$

much greater than the Newtonian value of gravity, but lower than the Coulombian force which is of the order of $10^{-7} \mathrm{~N}$.

\section{Emergent Newtonian Gravity}

The Equation (36) puts in evidence as gravity is produced by the conservation of the vacuum energy when the radius of the bubble, the wavelength of the DEMS, is stretched during the omega phase, i.e. when the wavelength of the direct interaction grows. To produce the gravity as a force between macroscopic body it is necessary to consider the strength between the particles with which the matter is formed. For example, a great number of hydrogen atoms are confined in a volume of space-time. Each proton and electron of the atoms interact electromagnetically with all the other particles of opposite charge. The resultant gravitational effects obtained adding up the fields emitted by each DEMS produced by the direct interaction between a positive and a negative charge, yields a resultant strength which value is varying with the energies localized by each DEMS and by their effective age in redshift terms. In this sense, if a DEMS is just formed on it does not act gravity. Using the generalized definitions (45) and (51), the total 
gravitational field produced by the particles is

$$
\Gamma=\sum_{i} Z_{i} \boldsymbol{g}_{i}
$$

Extending the Equation (58) to an arbitrary equal number $N_{p}=N_{e}=N_{H}$ of protons and electrons forming a cloud of hydrogen, the gravitational field acting on an observer is given by

$$
\Gamma=-G_{0} m_{p} \sum_{j=1}^{N_{P}} \frac{Z_{j}}{r_{j}^{2}} \hat{\boldsymbol{r}}_{j}-G_{0} m_{e} \sum_{k=1}^{N_{e}} \frac{Z_{k}}{r_{k}^{2}} \hat{\boldsymbol{r}}_{k}
$$

Since a bounded couple proton-electron is a hydrogen atom, using its spatial average position $r_{i}$ of the atom respect to the observer, Equation (59) becomes

$$
\Gamma=-G_{0}\left(m_{p}+m_{e}\right) \sum_{i=1}^{N_{H}} \frac{Z_{i}}{r_{i}^{2}} \hat{r}_{i}
$$

where considering the effective distance (46) of the observer from the moving center of mass of the cloud, is possible to define a mean function of the factors as

$$
\sum_{i=1}^{N_{H}} \frac{Z_{i}}{r_{i}^{2}}=\frac{1}{r^{2}} \sum_{i=1}^{N_{H}} Z_{i} \cos ^{2} \chi_{i}=\frac{N_{H} \bar{Z}}{r^{2}}
$$

where $\chi_{i}$ defines the angular distance of each atom from the center of mass of the cloud.

Equation (60) becomes

$$
\Gamma=-G \frac{M_{\text {cloud }}}{r^{2}} \hat{\boldsymbol{r}}
$$

where

$$
M_{\text {cloud }}=N_{H}\left(m_{p}+m_{e}\right),
$$

is the total mass of the hydrogen cloud, $G=G_{0} \bar{Z}$ is the Modified Gravitational Constant (MGC) as defined in Equation (53) where $\bar{Z}$ is the average ratio (51) calculated on all the hydrogen atoms. The gravity is produced from a spatial concentration of energy as an emergent real force which the effective strength depends not only from the mass and by the effective distance (46) but also from the effective MGC value which modulates the classical value of Newtonian gravity. Using the definition of effective distance (43), the redshift produced by the expansion of the bubble of space-time can be defined as

$$
z=\zeta_{B G}+\frac{2 r}{(1+b) \lambda_{b}}
$$

in agreement with (51) the scalar dimensionless ratio becomes

$$
Z=\frac{r}{\lambda_{b}}+\zeta_{B G}(1+b)
$$

with which using the gravitational force (62), the gravitational potential of a cloud of mass (63) becomes

$$
\Phi=-G_{0}\left[\lambda_{b}^{-1}+\frac{\zeta_{B G}(1+b)}{r}\right] M_{\text {cloud }} \hat{\boldsymbol{r}} .
$$


The consequences on large scale of the MGC in the Equation (66) can be much relevant, in fact considering two galaxies moving away at relativistic velocity, they are associated to a radial redshift $z=\beta$ with $\varphi \cong \pi / 2$ and will show a gravitational attraction greater than the one previewed by the Newtonian gravitational law as if their mass was greater than of the visible one. In this case, the gravitational anomaly is able to simulate the presence of an exceeding mass. Conversely, two nearby interacting galaxies, with a relative not relativistic motion $\beta \cong 0$, would show a gravity equal the one expected considering their visible mass. This effect, if confirmed, would eliminate the need for Dark Matter in the galactic halos

\section{Anomalous Rotational Velocity of Galaxies}

Another interesting effect usually attributed to Dark Matter could be observed considering the matter orbiting around a gravitational attractor, like the case of stars or gas orbiting around a galactic nucleus or a black hole. Since the motion occurs tangentially with $\varphi \cong 0$ around the attractor, the orbital velocity of matter is directly proportional to the square root of the absolute value of the gravitational potential of the attractor. In this case, considering the Equation (66) is possible immediately to write

$$
V_{r}=\sqrt{\left(\frac{G_{0}}{\lambda_{b}}+\frac{G}{r}\right) M}
$$

Since the Equation (67) has got a velocity constant term, can be rewritten in the form

$$
V_{r}=\sqrt{V_{0}^{2}+G \frac{M}{r}}
$$

where

$$
V_{0}=\sqrt{\frac{G_{0}}{\lambda_{b}} M}
$$

can be interpreted as the anomalous rotational velocity of the matter orbiting at great distance from the center of mass and it is coinciding with the bubble contribute at the velocity of the halo. The effect is a flat velocity of the galactic halo, so considering a galaxy, its external rotational velocity is a constant value (69) depending only by the mass contained within the spherical shell centered in the center of mass and by the value of the wavelength $\lambda_{b}$ of the redshifted gravitational wave associated at the maximum extension of the bubble of space-time containing the attractor, i.e. the wavelength $\lambda_{b}$ is the radius of the local universe connected gravitationally with the galaxy.

In general, considering the modified gravitational field (66) which produces a strength acting on the rotating matter much intense than the Newtonian one, the squared of the velocity of rotation in the Newtonian approach gives a mass much greater than what it really is 


$$
M_{\text {real }}=M_{\text {Newton }}-\frac{V_{0}^{2}}{G} r .
$$

In other terms, the orbital velocity gives an estimation of the Newtonian gravitational mass of an attractor greater than the one expected with the present theory. This fact provides a bubble associated with a galaxy with an effective mass energy formally in accordance with that estimated for an effective DEMS in Equation (37), in which a part of the initial energy became energy of the nothingness field which increases the tension of space-time of the galactic bubble

$$
E^{\prime}=E-\frac{c^{2} V_{0}^{2}}{G} r
$$

\section{The Einstein Field Equation for the Multi-Bubble Universe}

The gravity (54) is origined by the curvature of the local space-time of a DEMS produced by a concentration of energy inside the spherical wavefront but also from the relativistic motion of the two interacting observers which modifies the energy density in space-time. In this sense gravity is the final result of the interaction of two observers due to energy conservation of two particles electromagnetically entangled. To calculate the local curvature of the space-time of a single DEMS respect the centre of mass of the source, it is necessary to consider the total energy of the vacuum defined by the Equation (1). Defining the derivative of the volume of the first wavefront of the DEMS with respect the wavelength measured in the center of mass

$$
\frac{\mathrm{d} V}{\mathrm{~d} \lambda_{0}}=4 \pi \lambda_{0}^{2}
$$

and using Equation (72) and the strength (5) respect the center of mass, the scalar value of the energy density is

$$
T=\frac{\mathrm{d} U}{\mathrm{~d} V}=-\frac{h c}{4 \pi \lambda_{0}^{4}}+\frac{\sigma_{w}}{\lambda_{0}^{2}}
$$

Using the Equation (30) it is easy to obtain

$$
\frac{8 \pi G_{0}}{c^{4}} T=\frac{2}{\lambda_{0}^{2}}-\frac{1}{2} \frac{G_{0} h}{c^{3}}\left(\frac{2}{\lambda_{0}^{2}}\right)^{2}
$$

where the left side, the Equation (74) is the track of the Einstein's tensor

$$
\frac{8 \pi G_{0}}{c^{4}} g^{\mu v} T_{\mu v}=g^{\mu v} R_{\mu v}-\frac{1}{2} g^{\mu v} g_{\mu v} R .
$$

Since the track of the Ricci's tensor in Equation (75) is the scalar curvature $R$ and for a four-dimensional space-time $g^{\mu v} g_{\mu v}=4$, the Equation (75) can be rewritten in scalar terms as

$$
\frac{8 \pi G_{0}}{c^{4}} T=-R
$$

consequently, from the Equation (74) and (76), by using the BG energy (31) and 
the definition (41), the scalar curvature of a DEMS is

$$
-R=\frac{2}{\lambda_{0}^{2}}\left(1-\zeta_{B G}\right)
$$

Equation (77) shows how for $\zeta_{B G}=1$, corresponding to a gravitationally collapsed DEMS, the scalar curvature of space-time is zero as in a universe with zero energy density. This occurs because all the energy of the DEMS is transferred by means of the exchange of a BG to the energy of the field of nothingness, which behaves like a potential energy of space-time. The final effect is to maintain unchanged the total energy flattening the space-time. Vice versa in the case $\zeta_{B G} \ll 1$, the Equation (77) converges to the scalar curvature of the bubble of space-time with a radius equal to the wavelength of a DEMS referred to the own center of mass

$$
-R \cong \frac{2}{\lambda_{0}^{2}}
$$

Equation (78) corresponds to the curvature of the Beltrami pseudosphere with radius equal to the wavelength of the DEMS.

By using Equations (75), (76), the Equation (78) becomes

$$
-R=g^{\mu v} R_{\mu v}-\frac{1}{2} g^{\mu v} g_{\mu v} R
$$

and the Equation (77) can be rewritten in good approximation in the form

$$
\frac{8 \pi G_{0}}{c^{4}} g^{\mu \nu} T_{\mu v}=g^{\mu v} R_{\mu v}-\frac{1}{2} g^{\mu v} g_{\mu v} R+\frac{1}{4} \zeta_{B G} g^{\mu v} g_{\mu v} R
$$

which, in space-time with $0<\zeta_{B G} \ll 1$ is in agreement with the track of the Einstein's tensor (75) for a flat universe, associate to a close to zero cosmological term.

Equation (80) puts in evidence as the cosmological term is not a constant because it is varying with the amount of energy of the vacuum. In fact, considering a bubble with $\zeta_{B G}=1$, the track of the field Equation (80) and the scalar curvature (77) yield a null vacuum tension $T=0$ in an empty and flat bubble in which all energy is possessed by the field of nothingness. In each other case, the tensor associated to the field Equation (80) yields:

$$
\frac{8 \pi G_{0}}{c^{4}} T_{\mu \nu}=R_{\mu \nu}-\frac{1}{2} g_{\mu \nu} R+\frac{1}{4} \zeta_{B G} g_{\mu \nu} R
$$

where the third term on the right side corresponds to the energy of vacuum produced inside the DEMS, i.e. the cosmological term is a variable.

\subsection{Energy and Curvature of the Space-Time of a DEMS}

In terms of electromagnetic wave energy density, applying the definition of generalized Compton wavelength given in [8]

$$
\lambda_{0}=\frac{2 h c}{\varepsilon} \gamma(1-\beta \cos \theta)
$$


the scalar curvature (78) can be rewritten in the relativistic form

$$
R=-\frac{\varepsilon^{2}}{2 h^{2} c^{2}} \frac{1-\beta^{2}}{(1-\beta \cos \theta)^{2}}
$$

which contains the energy of the photon exchanged between the two interacting particles, namely of the photon emitted by a DEMS towards an observer. The Equation (83) highlights how space-time curvature depends on the square of the rest energy located within the DEMS, but also on the dynamic conditions in which the interaction occurs. In fact, the maximum of the curvature is achieved during the phase of approaching of the two interacting particles. In this case, the incidence angle is $\theta \cong 0$, using the definition of entangled energy (2.A), the Equation (83) becomes

$$
R=-\frac{\varepsilon_{1} \varepsilon_{2}}{2 h^{2} c^{2}} \frac{1+\beta}{1-\beta}
$$

in which for effect of the Doppler factor the curvature of the bubble increases during a relative high energy approach with $\beta \rightarrow 1$, but it is zero when in space-time, there is not electromagnetic energy density. Using Equation (84) in agreement with Equation (78) is possible to define the energy of the interaction as a function of the local curvature,

$$
E=h c \sqrt{-\frac{R}{2}}=\gamma \frac{\varepsilon}{2}(1+\beta)
$$

Equation (84) shows how curvature is a relativistic quantity, in fact, identical observers with different speeds feel different curvatures in presence of the same amount of energy. Following this idea for macroscopic bodies, the galaxies of a same cluster can be attracted to their common center of mass with different values of gravity, for effect of the difference among their masses but also by the different relative speed of each galaxy respect the center of mass. Speedy galaxies will experience high value of space-time curvature.

\subsection{The Einstein's Tensor and the Cosmological Term for a Multi-Bubble Universe}

To consider a whatever sphere of space-time in the universe with an arbitrary radius $r$ and not a single DEMS of wavelength $\lambda_{0}$, but a region in which many bubble can coexist in a space-time foam, using the Equations (5), (73) and (30) in the form

$$
\frac{\mathrm{d} V}{\mathrm{~d} \lambda_{0}} T=-\frac{h c}{\lambda_{0}^{2}}+\frac{c^{4}}{G_{0}}
$$

that considering the derivative of the volume of the multi-bubble can be rewritten as

$$
\frac{\mathrm{d} V}{\mathrm{~d} r} \frac{\mathrm{d} r}{\mathrm{~d} \lambda_{0}} T=-\frac{h c}{\lambda_{0}^{2}}+\frac{c^{4}}{G_{0}}
$$

Using the Equation (B.7) in Appendix B, Equation (87) becomes 


$$
\frac{8 \pi G_{0} Z}{c^{4}} T=\frac{2}{r^{2}}\left(1-\frac{G_{0} h}{c^{4}} \frac{1}{\lambda_{0}^{2}}\right)
$$

Using the definition of MGC and the definition (41), the Equation (88) can be rewritten as

$$
\frac{8 \pi G}{c^{4}} T=\frac{2}{r^{2}}\left(1-\zeta_{P}\right)
$$

where the effective gravitational pseudo-constant $G=G_{0} Z$ depends by the space-time dynamics. Similarly, to what was done for the field Equation (81), the Equation (89) gives the Einstein's field equation for a spherical region of any radius

$$
\frac{8 \pi G}{c^{4}} T_{\mu v}=R_{\mu v}-\frac{1}{2} g_{\mu v} R+g_{\mu v} \Lambda
$$

Considering the cosmological term at right hand of the Equation (90) its value is due to the nothingness field and as in Equation (89) decreases with the inverse of the squared of the distance from the center of mass of a whatever gravitational attractor, varying in time throughout the $\zeta_{B G}$ value, representing in the Equation (89) the Planck's antigravity-gravity threshold. This effect is justifying in peculiar conditions a rapid expansion of the considered multi-bubble universe without the necessity to suppose the existence of Dark Energy.

Assuming the nowadays value of $\zeta_{B G}$, the scalar field is estimated to be for great distances in agreement with a positive near zero cosmological term

$$
\Lambda=\frac{\zeta_{B G}}{2 r^{2}} \simeq \frac{3.8 \times 10^{-33}}{r^{2}} \approx 0
$$

decreasing with the squared of the radius $r$ of the sphere, delimiting a generic space-time multi-bubble. The Equation (91) in the present era emulates the presence of a very low positive dark energy able to change locally with the redshift value respect the threshold of $\zeta_{B G}$, producing in the multi-bubble contractive or expansive effects as predicted in the Equation (42). Considering the results given by the Planck Collaboration which given a measured Hubble constant value lower than $70 \mathrm{~km} \cdot \mathrm{s}^{-1} \cdot \mathrm{Mpc}^{-1}$ [15] and other measures performed on standard candles [16], gravitational lenses [17] and direct parallax [18] that give measures greater than the same value, to exemplify, assuming $H_{0} \approx 70 \mathrm{~km} \cdot \mathrm{s}^{-1} \cdot \mathrm{Mpc}^{-1}$ the Equation (91) yields

$$
\Lambda=\frac{\zeta_{B G} H_{0}^{2}}{2 c^{2}} \approx 10^{-85} \mathrm{~m}^{-2} .
$$

\section{Prediction and Structure of the Universe}

To adapt the Multi-Bubble model to the real observed universe is essential to choose observational data relevant for this model. Is possible to consider the universe from the human point of view, i.e. from the Earth center. Each spacecraft observation can be considered made from a lab placed in the Earth center, so the infrared cosmic background corresponds to the emission of the surface of the 
early universe that evolving stretches the primordial wavelength of the first bubble to the actual radius. Inside the universe, the action fluctuations produce new bubbles of space-time by expanding the primordial bubble originating the energy and the matter from which galaxies have taken origin. Considering this scenery is possible to place each galaxy on an ideal concentric spherical shell with a radius characterized by a peculiar value of redshift, so galaxies with equal redshift are positioned on the same ideal surface. Throughout the exchange of an integer number $N$ of BG, the radii of each concentric sphere grows adiabatically in time of an amount $\delta \lambda=2 N \lambda_{B G}$ increasing the redshift of a corresponding amount estimated by the Equation (27) until the external shell becomes coinciding with the visible cosmic microwave background (CMB).

\subsection{Estimation of the Amounts of Dark Energy, Dark Matter and Baryonic Matter Inside a Multi-Bubble Universe: Can $G_{B G}$ Be a Kaluza-Klein Graviton $G_{K K}$ ?}

Considering the current $\mathrm{CMB}$, what is observed from Earth can be considered to correspond to the spectral emission of the primordial bubble now redshifted towards the microwave electromagnetic spectrum. Before the creation of space-time, the nothingness field it exchanges energy with the electromagnetic field of vacuum through the exchange of BG mediators. The characteristic energy of a BG is estimated with the Equation (32) using the value of $G_{0}$ by resulting to be $E_{B G} \cong 2.68 \mathrm{TeV}$ (see Figure 1) perfectly in agreement with the mass energy limit of the Kaluza-Klein graviton $G_{K K}$ observed in the channel $G_{K K} \rightarrow l l$ by the ATLAS collaboration [19].

It is necessary to remember that the BG is a spin-zero boson with the characteristics of a gravitationally self-collapsed DEMS, its radius is under the own EH and its energy is formally equal to that of Planck in which the value of the gravitational constant $G$ is substituted by the primordial gravitational constant $G_{0}$, i.e. its energy is considered a primordial pseudo Planck energy.

Considering each possible spherical bubble, if its radius is smaller or equal than the Schwarzschild radius of a BG: $r \leq 2 \lambda_{B G}$ with $\lambda_{B G} \cong 4.62 \times 10^{-19} \mathrm{~m}$, the bubble cannot be visible because it is ever under the own EH $r_{E H} \approx 10^{-18} \mathrm{~m}$. Considering the condition (42-c), each graviton decaying produces a DEMS with a bubble influenced by antigravity only if its radius is within the range $\lambda_{B G} \leq r \leq 1.4656 \lambda_{B G}$ or by gravity if $r>1.4656 \lambda_{B G}$. In both cases each bubble with a radius in the region $1.4656 \lambda_{B G}<r \leq 2 \lambda_{B G}$ is not observable because collapsed below the $\mathrm{EH}$ but become observable when $r>2 \lambda_{B G}$.

As examined in paragraph 3.1, if the vacuum is in the ground state with $n=1$ no exchange of energy occur $(N=0)$, i.e. the energy of the vacuum remains unchanged, the radius of the bubble is steady and equal to $\lambda_{B G}$. Because the electromagnetic field and space-time do not exist externally, the black-body temperature in a not existing space-time is not defined and can be considered identically equal to zero. All the BG in the ground state are a condensate of $\mathrm{MBH}$ gra- 
vitationally active as discussed above, in order to the validity of the condition (42-c) but not visible because under their $\mathrm{EH}$ with radius of the order of $10^{-18} \mathrm{~m}$. They represent the natural limit of the universe because externally to their radius space-time does not exist. Considering as zero time the BG decay into a DEMS in a gravity range $r>1.4656 \lambda_{B G}$, from that moment the radius of the BG it expands becoming an electromagnetic bubble with the emission of a high energy pair lepton-lepton or gamma-gamma, during the DEMS evolution three distinct zones with different range of radius and energy can be considered:

\section{1) The Events Horizon Zone}

Each BG is a MBH with Compton wavelength $\lambda_{B G}$ associated to a DEMS with empty source zone defined by the spherical crown within the interval $\lambda_{B G} \leq 2 \mathrm{r} \leq$ $3 \lambda_{B C}$ 2. If only fluctuations of action with $n=1$ occur, the BG remain in the form of a non-observable condensate because under the own EH but gravitationally active for the own energy. The radius of the $\mathrm{G}_{\mathrm{BG}}$ is of the order of the internal limit of the source zone of the DEMS: $r_{B G}=\lambda_{B G} / 2$.

\section{2) The Expansion Zone}

When in the condensate fluctuations with $n>1$ occur, the initial wavelength $\lambda_{i}=(\sqrt{n}-\sqrt{n-1}) \lambda_{B G}<\lambda_{B G}$ is instantaneously adiabatically converted expanding the non-observable DEMS up to the final value of wavelength $\lambda_{f}=(\sqrt{n}+\sqrt{n-1}) \lambda_{B G}>2 \lambda_{B G}$ changing the energy of the DEMS but not the total energy of the space-time bubble. The energy of the original fluctuation it can be converted only in space-time beyond the events horizon producing in the bubble a mean Planck's spectrum temperature greater than zero. Thus, an observer positioned in space-time outside the $\mathrm{EH}$ sees the emission of pairs of particles or of pairs of photons only from a minimum distance $r>2 \lambda_{B G}$. The energy of the initial fluctuation evolves under form of a real DEMS with stretched wavelength $\lambda_{f}>2 \lambda_{B G}$ beyond the events horizon. In this case, the BG exchanged between the electromagnetic field and the field of nothingness, decaying turns into a pair of particles that have the possibility to escape from each other or annihilate themselves by increasing in any case the energy of the visible universe.

\section{3) The Far Universe Zones}

After successive fluctuations, the universe is expanded and space-time grew up to become the current universe. The cosmic background observed today from a distance $r=c / H_{0}$ is the primordial events horizon in which it is possible to observe the primordial quantum fluctuations under form of energy and matter emissions from the Schwarzschild surface of the primordial condensate.

To estimate the percentage of Dark Energy, Dark Matter and ordinary matter observed in the universe it is necessary to calculate the value of the Planck's threshold characterizing the cosmic background formation in three different situations:

a) Dark Energy. Is represented by the effects on the observable universe of the energy $U_{0}$ of each condensate component corresponding to a fluctuation 
$n=1$. The total energy is not observable because is under the events horizon, but is gravitationally active both in producing antigravity and gravity acting on the matter in the actual universe. Using the expansion zone (2), the typical maximum length characterizing the quantum fluctuations among the $B G$ is $\lambda \cong \lambda_{B G} / 2$. The use of the Equation (41) gives a threshold $\zeta_{B G}^{(D E)} \cong 4$.

b) Dark Matter. Is represented by the all energy spectrum emitted beyond the lower limit of the source zone under the events horizon. To estimate the contribute of the Dark Matter it is necessary consider the primordial Planck's threshold (41) characterizing the energy emitted in the source zone under the events horizon of each BG. These energy and matter cannot be observable but acts gravitationally on all energy and matter placed in space-time beyond the events horizon. Since the wavelength of each DEMS corresponds to the minimal distance $R$ of interaction of a pair of particles emitted by the graviton during the decaying, the ratio $\rho=R / \lambda_{i}$ in the source zone of the DEMS is always within the interval $1<\rho \leq 3 / 2$. Using the structural mean ratio estimated accurately in $\mathrm{BT}$ : $\bar{\rho} \cong 1.276$, for a spectrum produced by a pair of interacting particles with minimum interaction distance $R \equiv \lambda_{B G}$, the Equation (41) gives $\zeta_{B G}^{(D M)}=\bar{\rho}^{2} \cong 1.63$ with an upper limit of 2.25 imposed by the external boundary of the source zone $1<\zeta_{B G}^{(D M)} \leq 9 / 4$.

c) Ordinary Energy and Matter. To estimate the energy associated to all the ordinary matter and electromagnetic energy of the bubble is necessary to calculate the Planck's threshold characterizing the energy that each DEMS emits in space-time, i.e. in the zone external to the $\mathrm{EH} \quad \lambda \geq r_{E H}=2 \lambda_{B G}$. Considering the energy emitted from the events horizon border towards space-time, the Equation (43) gives the threshold $\zeta_{B G}^{(M)} \cong 0.250$.

Considering the far universe zone (3), when the radius of the expanding bubble becomes the radius of the current multi-bubble universe and $r=c / H_{0}$, the far cosmic background observed is characterized by the overlapping of the three different effects described above. Using the definition of the ratio of the density of the universe to the critical density associated to the cosmological term:

$$
\Omega_{\Lambda}=\frac{1}{3}\left(\frac{c}{H_{0}}\right)^{2} \Lambda
$$

with the Equation (92) is possible to estimate the percentages of energies produced during the expansion of the multi-bubble universe. Denoting with a star the primordial value of the Planck's threshold

$$
\Omega_{\Lambda}^{*}=\frac{\zeta_{B G}^{*}}{6}
$$

assuming as radius of the universe the Hubble value, dividing in two parts the primordial Planck's threshold as defined in the previous points (a) and (b) $\zeta_{B G}^{*}=\zeta_{B G}^{(D E)}+\zeta_{B G}^{(D M)}$, Equation (94) yields

$$
\Omega_{\Lambda}^{*}=\frac{5.63}{6} \cong 0.938
$$


respectively $\Omega_{\Lambda_{D E}}^{*} \cong 0.667$ and $\Omega_{\Lambda_{D M}}^{*} \cong 0.271$ non-visible because under the events horizon.

On the other hand, the total amount of energy associated to the ordinary visible baryonic matter and energy $(C)$ is

$$
\Omega_{B}^{*}=\frac{\zeta_{B G}^{(B)}}{6}=\frac{0.250}{6} \cong 0.0417
$$

Considering the sum of the three components: Dark Energy, Dark Matter (95) and ordinary energy (96) (see Table 1 and Figure 3)

$$
\Omega^{*}=\Omega_{\Lambda}^{*}+\Omega_{B}^{*} \cong 0.980 \simeq 1
$$

the value (97) results lower than one, justifying the continued expansion weakly accelerated of the multi-bubble. A small positive or negative difference respect to $\Omega=1$ is probably due to the chosen value of the primordial Planck's threshold for the Dark Matter, perhaps more correctly a little larger than that estimated for the statistical effects can occur on the events horizon. Surely, however, considering the external value of the source zone of the bubbles the model limits the total omega value to be less than 1.083 .

Table 1. Omega values and percentages of energy and matter in the universe formation Estimated by the Multi-Bubble Model.

\begin{tabular}{ccc}
\hline & Percentage of Component & \multicolumn{2}{c}{ Density Ratio Contribute } \\
\hline Type of Component & $\Omega_{i}^{*} / \Omega^{*}(\%)$ & $\Omega^{*}$ \\
Dark Energy & 68.06 & 0.6667 \\
Dark Matter & 27.69 & 0.2712 (upper limit 0.3750) \\
Ordinary Matter and EM Energy & 4.25 & 0.0417 \\
Total & 100.00 & 0.9796 (upper limit 1.0834) \\
\hline
\end{tabular}

\section{Energy and Matter in the Multi-Bubble}

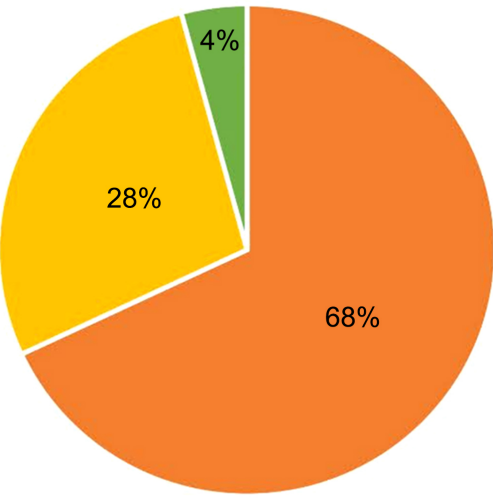

- Dark-Energy - Dark-Matter $\quad$ - Ordinary Energy and Matter

Figure 3. Distribution of energy and matter related to the index $\Omega_{i}^{*} / \Omega^{*}$ of Table 1 . 
Considering only the cosmological variable term, is possible to deduce that in the early universe on the $\mathrm{EH} \quad r=r_{E H}$ its value was close to

$$
\Lambda^{*}=\frac{\zeta_{B G}^{*}}{8 \lambda_{B G}^{2}} \simeq 3.4 \times 10^{+36} \mathrm{~m}^{-2}
$$

decreasing with the square of the temperature of the $\mathrm{CMB}$

$$
\Lambda=\frac{k_{b}^{2}}{8 h^{2} c^{2}} T^{2}
$$

at the current average value (92) close to zero. Equation (99) suggests as in different bubbles with different local temperatures the cosmological term can be different from the mean value (92), producing faster or slower expansions.

The recent measured values of omega are in very good agreement with the theoretically values estimated with the multi-bubble model presented in Table 1 .

\subsection{On the Estimation of the Actual Radius of the Gravitationally Active Universe}

The estimation of the anomalous rotation velocities of the galaxies implies the estimation of the length $\lambda_{b}$ characterizing the effective gravitational radius of the bubble of space-time in which the galaxy is been produced, i.e. the stretched value of the wavelength of the primitive bubble in which a galaxy has taken origin.

In order to analyze the sample of galaxies [20] in Table 2, all with a redshift very close to $z \sim 1$, i.e. all positioned on a spherical shell of radius equal to the distance of the galaxies from the observer, is necessary to consider to verify the linear relation between the squared of the flat velocity $Y$ of each galaxy with its mass $X$. The evidence of their linear proportionality $R_{1}: Y=\alpha_{11} X+\alpha_{01}$ is shown in Figure 4. The linear regression coefficients are: $\alpha_{11}=3.54 \times 10^{-37}$ $\mathrm{km}^{2} \cdot \mathrm{s}^{-2} \cdot \mathrm{kg}^{-1}$ and $\alpha_{01}=1.12 \times 10^{4} \mathrm{~km}^{2} \cdot \mathrm{s}^{-2}$ at $R^{2}=0.9314$. Considering Equation (68) and (69) in the form

$$
V_{f}^{2}=\frac{G}{r_{b}} M+V_{0}^{2}
$$

let

$$
\left\{\begin{array}{l}
\frac{G}{r_{b}}=\alpha_{1 j} \\
V_{0}^{2}=\frac{G_{0} M}{\lambda_{b}}=\alpha_{0 j}
\end{array}\right.
$$

to be the regression coefficients of the Equation (100), using the Equations (68) and (70) is possible to define for the galaxies sample in Table 2.

$$
\left\{\begin{array}{l}
\bar{V}_{0}=\sqrt{\alpha_{0 j}} \\
\frac{\bar{\lambda}_{b}}{M}=\frac{G_{0}}{\alpha_{0 j}} \\
\bar{r}_{b}=\frac{G}{\alpha_{1 j}} \\
\bar{H}_{b}=\frac{c}{\bar{\lambda}_{b}}
\end{array}\right.
$$


Table 2. Sample I: redshift $z_{r} \approx 1$.

\begin{tabular}{|c|c|c|c|c|c|c|c|}
\hline \multicolumn{8}{|c|}{ Observed Galactic Parameters and Estimations of the Characteristics of the Bubble } \\
\hline Galaxy & $\beta \equiv z_{r}$ & $\begin{array}{c}M_{g} \\
{\left[\times 10^{9} \mathrm{M}_{\mathrm{s}}\right]}\end{array}$ & $\begin{array}{c}M_{g} \\
{\left[\times 10^{39} \mathrm{~kg}\right]}\end{array}$ & $\begin{array}{c}V_{f} \\
{[\mathrm{~km} / \mathrm{s}]}\end{array}$ & $\begin{array}{c}V_{f}^{2} / M_{g} \\
{\left[\times 10^{-30} \mathrm{~km}^{2} \cdot \mathrm{s}^{-2} \cdot \mathrm{kg}^{-1}\right]}\end{array}$ & $\begin{array}{c}\lambda_{b} \\
{\left[\times 10^{51} \mathrm{~m}\right]}\end{array}$ & $\begin{array}{c}H_{b} \\
{\left[\times 10^{-24} \mathrm{~km} \cdot \mathrm{s}^{-1} \cdot \mathrm{Mpc}^{-1}\right]}\end{array}$ \\
\hline zmvvd_z1_87 & 0.896 & 3.31 & 6.59 & 96 & 1.40 & 6.20 & 1.49 \\
\hline zmus_z1_86 & 0.841 & 3.55 & 7.06 & 109 & 1.68 & 5.16 & 1.80 \\
\hline u3_5138 & 0.809 & 5.50 & 10.9 & 128 & 1.50 & 5.79 & 1.60 \\
\hline hiz_z1_258 & 0.838 & 5.62 & 11.2 & 117 & 1.22 & 7.09 & 1.31 \\
\hline zmus_z1_129 & 0.995 & 6.76 & 13.4 & 122 & 1.11 & 7.84 & 1.18 \\
\hline zmus_z1_125 & 0.998 & 8.91 & 17.7 & 141 & 1.12 & 7.74 & 1.20 \\
\hline zmus_z1_217 & 0.895 & 10,5 & 20.8 & 146 & 1.02 & 8.48 & 1.09 \\
\hline u3_25160 & 0.897 & 11.2 & 22.3 & 133 & 0.793 & 11.0 & 0.845 \\
\hline zmus_z1_166 & 0.975 & 12.9 & 25.6 & 148 & 0.855 & 10.2 & 0.912 \\
\hline u3_14150 & 0.896 & 12.9 & 25.6 & 144 & 0.809 & 10.7 & 0.863 \\
\hline zcos_z1_192 & 0.917 & 14.8 & 29.4 & 147 & 0.735 & 11.8 & 0.783 \\
\hline zmus_z1_119 & 0.839 & 21.9 & 43.5 & 179 & 0.736 & 11.8 & 0.785 \\
\hline zmus_z1_21 & 0.839 & 25.1 & 50.0 & 157 & 0.493 & 17.6 & 0.526 \\
\hline hiz_z1_258 & 0.838 & 25,7 & 51.1 & 173 & 0.585 & 14.8 & 0.624 \\
\hline zcos_z1_202 & 0.841 & 34,7 & 69.0 & 188 & 0.512 & 16.9 & 0.547 \\
\hline zcos_z1_690 & 0.927 & 38.9 & 77.4 & 208 & 0.559 & 15.5 & 0.596 \\
\hline zcos_z1_692 & 0.930 & 40.7 & 81.0 & 190 & 0.446 & 19.5 & 0.475 \\
\hline gs3_22005 & 0.954 & 52.5 & 104 & 215 & 0.443 & 19.5 & 0.472 \\
\hline Average & & 18.6 & 37.1 & 152 & 0.890 & 11.5 & 0.802 \\
\hline
\end{tabular}

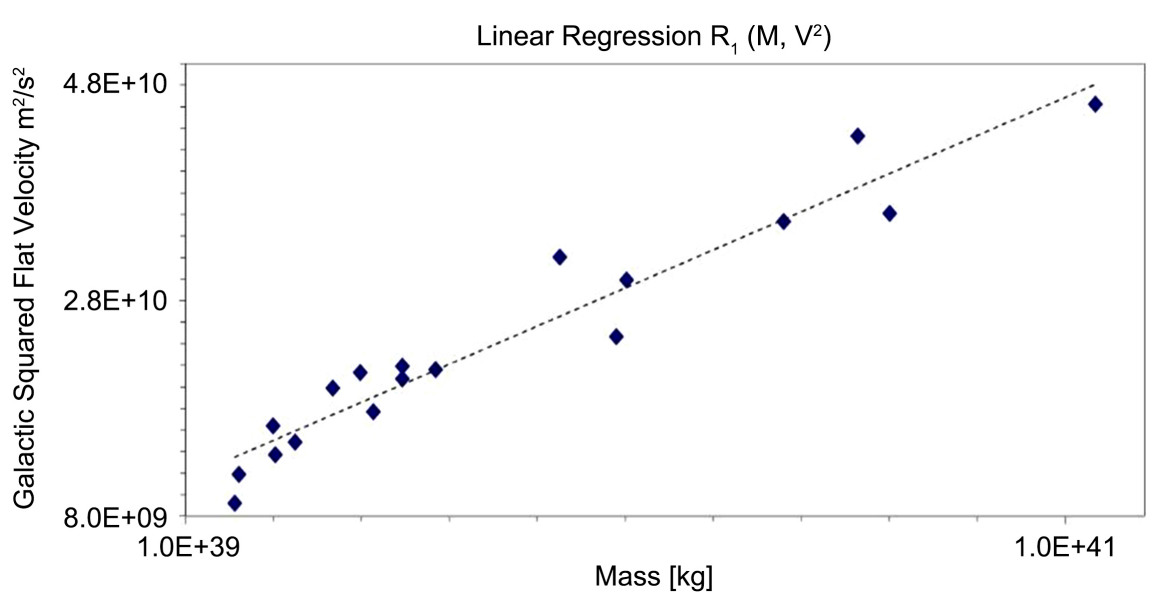

Figure 4. Linear regression of a sample of 18 galaxies with redshift $z \sim 1$. 
respectively the average flat velocity of the galaxy, the current average wavelength of the gravitational wave signal per unit of mass associated to a bubble, the average galactic radius of the active galactic haloes and the average Hubble constant of expansion of the bubble associated to the effective universe radius. Considering the Equation (55) and assuming a radial movement in which galaxies go away from the observer, assuming: $b=0$ and $z \cong 1$; using the values of $G_{0}$ and $G$ the Equation (101) gives

$$
R_{1} \rightarrow\left\{\begin{array}{l}
\bar{V}_{0} \simeq 110 \mathrm{~km} / \mathrm{s} \\
\bar{\lambda}_{b} / M \simeq 7.8 \times 10^{+8} \mathrm{~km} / \mathrm{kg} \cong 7.6 \times 10^{+20} \mathrm{Mpc} / \mathrm{M}_{\odot} \\
\bar{r}_{b} \simeq 1.9 \times 10^{+17} \mathrm{~km} \cong 6.1 \mathrm{kpc}
\end{array}\right.
$$

It is important to highlight as the Hubble constant of the bubble defined in the Equation (102) can be used to estimate the age of the first gravitational emission of the bubble, i.e. the elapsed time by the first quantum fluctuation which has produced the bubble in which the galaxy has taken origin. Its value is associated at the maximum extension of the bubble of space-time containing the galaxy, i.e. the wavelength $\lambda_{b}$ is the radius of the real universe connected gravitationally with the galaxy.

Using the values in column seven of Table 2, is possible to note how the radius of the space-time gravitationally linked to each galaxy in the sample is greater than the estimated radius of the universe, because the gravitationally linked bubble extension is proportional to the mass of the galaxy, that is, at the number of quantum fluctuations that within the bubble produced the mass energy of the galaxy. To prove the hypothesis of the gravitational connection of galaxies with a space-time bubble larger than the current estimation of the universe radius is enough to consider a second heterogeneous sample of galaxies [21] in Table 3. In this case, although the galaxies have different redshifts that make them not comparable in terms of belonging to the same spherical shell with the same gravitational constant value $G=G_{0} Z$, the linear regression analysis performed on the galactic rotation speed and mass data of the galaxies, shows an average behavior perfectly in accordance with the results of the previous sample in Table 2.

In fact, considering the linear regression law $R_{2}: Y=\alpha_{12} X+\alpha_{02}$ shown in Figure 5, the regression coefficients are: $\alpha_{12}=3.00 \times 10^{-36} \mathrm{~km}^{2} \cdot \mathrm{s}^{-2} \cdot \mathrm{kg}^{-1}$ and $\alpha_{02}=1.11 \times 10^{4} \mathrm{~km}^{2} \cdot \mathrm{s}^{-2}$ at $R^{2}=0.6462$, with which using the value $\alpha_{02}$

$$
R_{2} \rightarrow\left\{\begin{array}{l}
\bar{V}_{0} \simeq 110 \mathrm{~km} / \mathrm{s} \\
\bar{\lambda}_{b} / M \simeq 7.8 \times 10^{+8} \mathrm{~km} / \mathrm{kg} \cong 7.6 \times 10^{+20} \mathrm{Mpc} / \mathrm{M}_{\odot}
\end{array}\right.
$$

gives values totally in agreement with the results obtained with $R_{1}$ in Equation (103).

This unexpected but hoped-for compatibility between the two galaxies samples highlights a homology in galactic bubble structures relative to their age and extent in terms of gravitational interaction. Using Equation (55) and (68) 


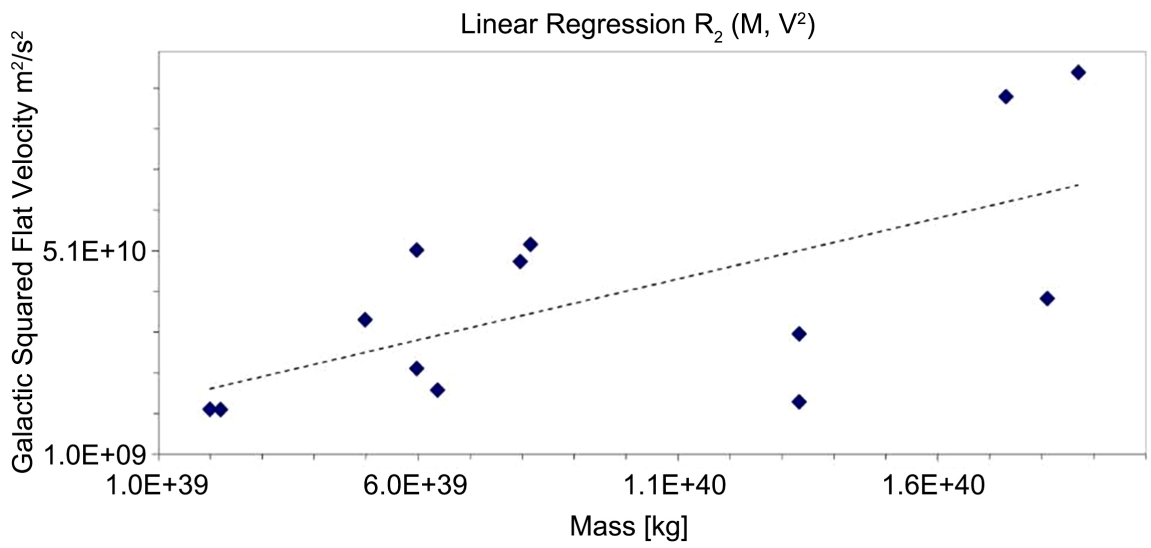

Figure 5. Linear regression of the sample II of 13 galaxies with different redshift.

$$
\lambda_{b}^{-1}=\frac{V_{0}^{2}}{M G_{0}}
$$

with which is possible to estimate $H_{b}^{-1}=\lambda_{b} / c$ which reciprocal is tabulated in last column of Table 2 and Table 3, yielding the age of the quantum fluctuation from which each bubble has taken origin. The reciprocal value $H_{b}$ is a characteristic Hubble constant of the bubble associated to the local redshift $z=H_{b} r / c \equiv r / \lambda_{b}$ as a function of the radius of a spherical shell inside the bubble giving the local Hubble law $v=H_{b} r$. While each quantum fluctuation is a boson, and in each bubble can be produced many fluctuations each producing a bubble, the primitive bubble expands like an overlap of bubbles with Hubble's law depending on the radius of the spherical shell considered

$$
v=\left(\sum_{j=1}^{k} H_{b j}\right) r=H_{0} r
$$

describing how the universe is seen expanding from an observer at any point in space-time. Following the pattern is evident that each bubble has a Hubble's expansion constant which is variable with the number of fluctuations in the field of nothingness from which its matter originated and with the uncertainty due to the accuracy of the measurements of the velocities of own galactic halo. Using the data of the sample of 13 galaxies in Table 3, the evaluation of the average gravitational wavelengths of the galactic bubbles and consequently of the average galactic Hubble's constant associated to the sample of galactic bubbles is

$$
\bar{H}_{b}=(5.1 \pm 2.4) \times 10^{-24} \mathrm{~km} \cdot \mathrm{s}^{-1} \cdot \mathrm{Mpc}^{-1}
$$

Considering the space-time as a foam in which each bubble contributes to the expansion pushing matter on the borders of the bubbles, is possible estimate the average number of bubbles, i.e. of active galaxies along the direction of observation in the universe as the ratio between the Hubble's constant value and the average galactic Hubble of the bubble (107), giving $k_{g}=H_{0} / H_{b}$ in such a way that the standard Hubble's constant is defined as

$$
H_{0}=\sum_{j=1}^{k_{g}} H_{b j}=k_{g} \bar{H}_{b}
$$


Table 3. Sample II, heterogeneous $z_{r}$

\begin{tabular}{|c|c|c|c|c|c|c|c|c|}
\hline \multicolumn{9}{|c|}{ Observed Galactic Parameters and Estimations of the Characteristics of the Bubble } \\
\hline Galaxy & Type & $\begin{array}{c}\beta \equiv z_{r} \\
{\left[\times 10^{-3}\right]}\end{array}$ & $\begin{array}{c}M_{g} \\
{\left[\times 10^{9} \mathrm{M}_{\mathrm{s}}\right]}\end{array}$ & $\begin{array}{c}M_{g} \\
{\left[\times 10^{39} \mathrm{~kg}\right]}\end{array}$ & $\begin{array}{c}V_{f} \\
{[\mathrm{~km} / \mathrm{s}]}\end{array}$ & $\begin{array}{c}V_{f}^{2} / M_{g} \\
{\left[\times 10^{-30} \mathrm{~km}^{2} \cdot \mathrm{s}^{-2} \cdot \mathrm{kg}^{-1}\right]}\end{array}$ & $\begin{array}{c}\lambda_{b} \\
{\left[\times 10^{51} \mathrm{~m}\right]}\end{array}$ & $\begin{array}{c}H_{b} \\
{\left[\times 10^{-24} \mathrm{~km} \cdot \mathrm{s}^{-1} \cdot \mathrm{Mpc}^{-1}\right]}\end{array}$ \\
\hline M33 & Scd & -0.60 & 1.0 & 1.99 & 110 & 6.10 & 1.42 & 6.51 \\
\hline UGC7321 & Sd & 1.38 & 1.1 & 2.19 & 110 & 5.49 & 1.58 & 5.85 \\
\hline NGC253 & $\mathrm{Sc}$ & 0.86 & 2.5 & 4.97 & $\approx 185$ & 6.86 & 1.26 & 7.32 \\
\hline NGC3044 & $\mathrm{Sc}$ & 4.30 & 3.0 & 5.97 & 149 & 3.71 & 2.34 & 3.95 \\
\hline M31 & $\mathrm{Sb}$ & -1 & 3.0 & 5.97 & 226 & 8.58 & 1.01 & 9.15 \\
\hline NGC2403 & Scd & 0.43 & 3.2 & 6.36 & 130 & 2.65 & 3.28 & 2.83 \\
\hline Milky Way & $\mathrm{Sb}$ & - & 4.0 & 7.96 & 220 & 6.08 & 1.43 & 6.49 \\
\hline NGC891 & $\mathrm{Sb}$ & 1.76 & 4.1 & 8,15 & 220 & 6.46 & 1.34 & 6.89 \\
\hline NGC4559 & Scd & 2.72 & 6.7 & 13.3 & 118 & 1.05 & 8.30 & 1.12 \\
\hline NGC6946 & Scd & 0.15 & 6.7 & 13.3 & 175 & 2.30 & 3.78 & 2.45 \\
\hline NGC2613 & $\mathrm{Sb}$ & 5.61 & 8.7 & 17.3 & $\approx 298$ & 5.14 & 1.71 & 5,48 \\
\hline NGC5775 & $\mathrm{Sb}$ & 5.67 & 9.1 & 18.1 & 198 & 2.17 & 4.00 & 2.32 \\
\hline NGC5746 & $\mathrm{Sb}$ & 5.75 & 9.4 & 18.7 & 308 & 5.08 & 1.71 & 5.41 \\
\hline Averages & & & & & & 4.74 & 2.55 & 5.06 \\
\hline
\end{tabular}

In Figure 6 and Figure 7 are plotted the values of the galactic Hubble constant for the two samples of galaxies used. Since the Equation (108) implies that the Hubble constant is not a true constant, because it depends on the random number of gravitationally active bubbles (primordial fluctuations) in the space-time zone in which the measurement is performed, the universe expands unevenly, and measurements obtained using different astrophysical methods in different regions of space-time can yield different results, requiring complex reinterpretations of the data [22].

As an example considering a measure performed on a space-time zone of the universe giving $H_{0}=75 \mathrm{~km} \cdot \mathrm{s}^{-1} \cdot \mathrm{Mpc}^{-1}$, assuming the average value of $\bar{H}_{b}=(5.06 \pm 2.4) \times 10^{-24} \mathrm{~km} \cdot \mathrm{s}^{-1} \cdot \mathrm{Mpc}^{-1}$ in Table 3 is possible to estimate the approximate number of bubbles, i.e. the number of the gravitational active local bubbles as ratio between the value of the Hubble's constant and the average galactic Hubble constant of the primordial local bubble as $k_{g}=H_{0} / \bar{H}_{b} \approx 1.5 \times 10^{25}$. This consideration suggests that in volumes of space-time with high density of energy and matter, a large number of primordial fluctuations were produced from the origin at the current time, each producing a bubble with energy and matter currently largely structured in galaxies, in this case, the Hubble constant may be greater than that measured in a space-time with a lower density of energy and matter, that is, the measurement of Hubble's constant is directional and depends on the time difference separating the space-time observed. 


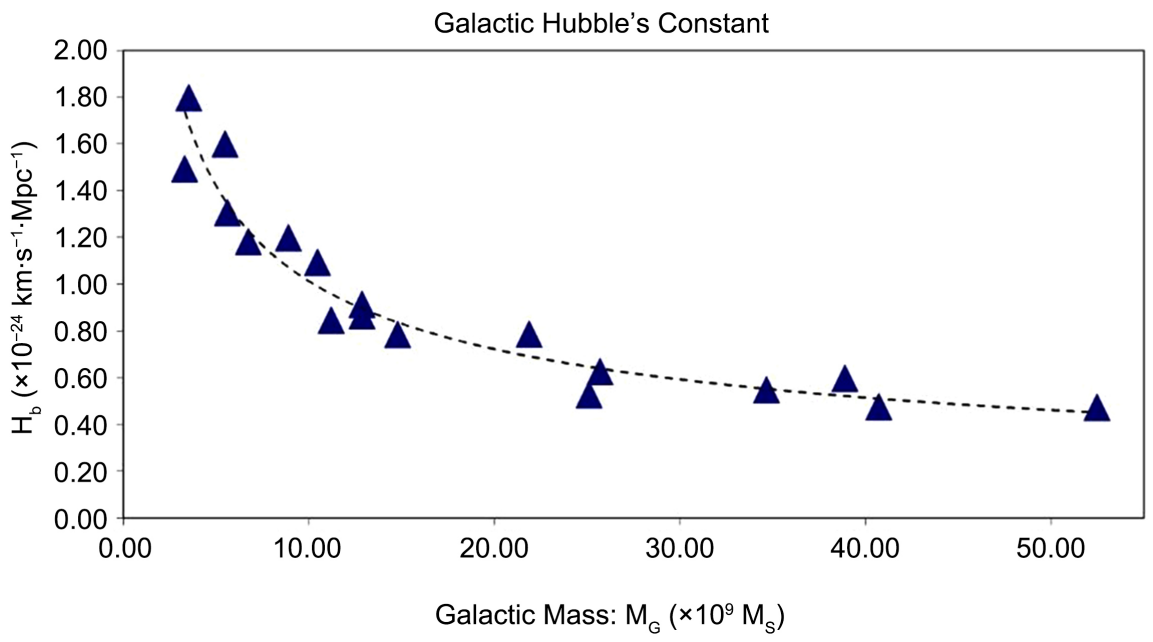

Figure 6. Galactic Hubble expansion constant associated to the sample one of galaxies as a function of the mass.

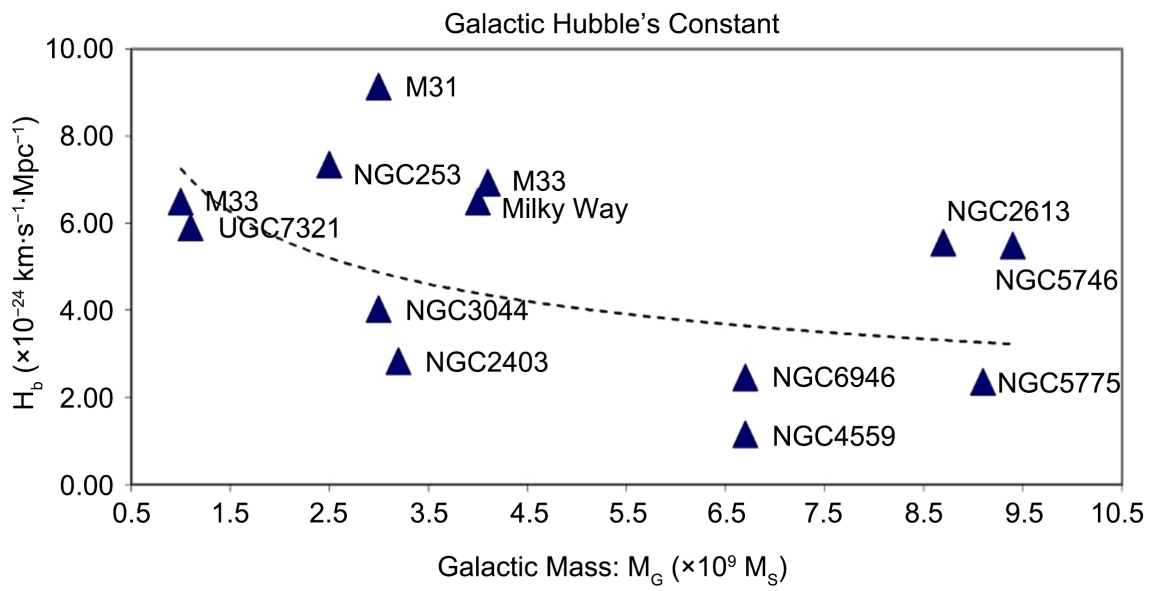

Figure 7. Galactic Hubble expansion constant associated to the sample two of galaxies as a function of the mass.

\section{Conclusions}

In this work, a gravitational theory has been developed within a space-time model based on the foundations of Bridge Theory, a theory that unifies quantum-relativistic phenomenologies and electro-gravitational forces. In fact, this model has strong roots in quantum theory and relativity theory, a way to rethink Maxwell's Electromagnetic Theory as the common basis of the entire physical world.

The model uses the notion of DEMS and describes space-time as a foam in which each bubble is a Dipole Electromagnetic Source (DEMS) that exchanges Balancing Gravitons (BG) with the potential energy of the vacuum. The exchange of BG, estimated to correspond in mass energy to Kaluza-Klein gravitons of $2.68 \mathrm{TeV}$ observed in Run \#1 in ATLAS, it helps to increase the electromagnetic energy and matter that powers galaxies in a spherical bubble of space-time, just a brick of the universe. The spontaneous creation of new bubbles keeps in 
balance the negative and positive pressure in space-time that expands differently zones of universe according to the number of bubbles produced inside. The resulting effect leads the Hubble constant to have different values with direction without this implying the existence of a real Dark Energy in play, but only the presence of accelerations produced by the variability of the number of the expanding bubbles, i.e. by the variability of the distribution of the galaxies along the sight line of the observer. The dynamic evolution of each DEMS induces a local curvature of space-time that characterizes each bubble, so gravity acts on the whole matter within the bubble. Considering the universe as a foam of DEMS, gravity is proved to transcend the boundaries of the bubble acting on all matter of the universe in accordance with Einstein's field equation but with a modified gravitational constant changing value with a set of variables depending on the dynamics of the direct interaction and on the energy of the bubble. Within the bubbles, the Newtonian strength is modified by the continued change in the scale factor producing at great distances a more intense gravity. The resulting effect justifies abnormal rotations of galactic halos without using Dark Matter in the form of unknown particles or mechanically unjustified MOND theories, because it is the energy of the vacuum hidden under the fabric of the universe that acts gravitationally. In terms of General Relativity, the model is in accordance with the presence of a not constant lambda cosmological term nowadays very close to zero and of an omega term very close to one but not necessarily everywhere equal.

\section{Conflicts of Interest}

The author declares no conflicts of interest regarding the publication of this paper.

\section{References}

[1] Rees, M.J. (2003) Philosophical Transactions of the Royal Society of London. Series A: Mathematical, Physical and Engineering Sciences, 361, 2427-2434. https://doi.org/10.1098/rsta.2003.1289

[2] Di Valentino, E., Melchiorri, A. and Silk, J. (2019) Nature Astronomy, 4, 196-203. https://doi.org/10.1038/s41550-019-0906-9

[3] Abbott, B.P., Abbott, R., Abbott, T.D., Acernese, F., Ackley, K., Adams, C., Adams, T., Addesso, P., Adhikari, R.X., Adya, V.B., et al. (2017) Astrophysical Journal Letters, 851, L35.

[4] GRAVITY Collaboration (2018) Astronomy \& Astrophysics, 615, L15.

[5] Auci, M. and Dematteis, G. (1999) International Journal of Modern Physics B, 13, 1525-1557. https://doi.org/10.1142/S0217979299001569

[6] Auci, M. (2018) Journal of Modern Physics, 9, 2206-2222. https://doi.org/10.4236/jmp.2018.912139

[7] Auci, M. (1990) Physics Letters A, 148, 399-404. https://doi.org/10.1016/0375-9601(90)90488-A

[8] Auci, M. (1990) Physics Letters A, 150, 143-150. 
https://doi.org/10.1016/0375-9601(90)90109-2

[9] Auci, M. (1990) Physics Letters A, 135, 86-88.

https://doi.org/10.1016/0375-9601(89)90650-6

[10] Auci, M. (2020) SSRG International Journal of Applied Physics, 7, 7-15. https://doi.org/10.14445/23500301/IJAP-V7I2P102

[11] Auci, M. (2006) Self-Gravitational Red Shift Effect and Micro-Black Holes Production in Dipolar Electromagnetic Sources.

[12] Estakhr, A.R. (2016) APS Annual Meeting of the Far West Section Vol. 61, Num. 17.

[13] Prevedelli, M., Cacciapuoti, L., Rosi, G., Sorrentino, F. and Tino, G.M. (2014) Philosophical Transactions of the Royal Society A, 372, Article ID: 20140030. https://doi.org/10.1098/rsta.2014.0030

[14] Auci, M. (2008) Fundamentality of the Sommerfeld's Fine Structure Constant in Bridge Theory and Consequences on the Fundamental Atomic Constants.

[15] Aghanim, N., et al. (2020) A\&A, 641, A6. https://doi.org/10.1051/0004-6361/201833910

[16] Amendola, L. and Quartin, M. (2019) Measuring the Hubble Function with Standard Candle Clustering.

[17] Jee, I., et al. (2019) Science, 365, 1134-1138. https://doi.org/10.1126/science.aat7371

[18] Croft, R.A.C. (2020) Direct Geometrical Measurement of the Hubble Constant from Galaxy Parallax: Predictions for LSST/VRO and WFIRST.

[19] Aad, G., et al. (2014) Physical Review D, 90, Article ID: 052005.

[20] Di Teodoro, E.M., Fraternali, F. and Miller, S.H. (2016) Astronomy \& Astrophysics, 594, A77. https://doi.org/10.1051/0004-6361/201628315

[21] SIMBAD, Astronomical Data Base.

[22] Verde, L., Treu, T. and Riess, A.G. (2019) Nature Astronomy, 3, 891-895. https://doi.org/10.1038/s41550-019-0902-0 


\section{Appendix A: Definition of Entangled Masses}

From Ref. [6] the relativistic energy of the interacting particles forming a DEMS at rest is

$$
\varepsilon=\sqrt{\left(E_{1}+E_{2}\right)^{2}-\left\|\boldsymbol{P}_{1}+\boldsymbol{P}_{2}\right\|^{2} c^{2}}=\sqrt{\varepsilon_{1}^{2}+\varepsilon_{2}^{2}+2 \varepsilon_{1} \varepsilon_{2} \gamma_{1} \gamma_{2}\left(1-\beta_{1} \beta_{2} \cos \vartheta\right)}
$$

where $\vartheta$ is the angle between the momenta $\boldsymbol{P}_{1}$ and $\boldsymbol{P}_{2}$ of the two colliding particles with respect to the lab-frame $S$. Equation (A.1) can be also considered equal to a mixed rest energy of the two interacting particles in such a way the squared rest energy can be expressed as the product of two entangled mass energy terms

$$
\varepsilon^{2}=4 \mu_{1} \mu_{2} c^{4}
$$

each defining the entangled rest mass of one of the two particles

$$
\mu_{i}=\frac{\varepsilon_{i}}{2 c^{2}} \sqrt{\frac{\varepsilon_{1}}{\varepsilon_{2}}+\frac{\varepsilon_{2}}{\varepsilon_{1}}+2 \gamma_{1} \gamma_{2}\left(1-\beta_{1} \beta_{2} \cos \vartheta\right)} \quad i=1,2
$$

in fact, the observer cannot distinguish the rest energies of a single interacting particle because each particle is entangled with the other or with the observer with which it produces the DEMS. For two identical interacting particles with same rest energy $\varepsilon_{1} \equiv \varepsilon_{2}=\varepsilon$ if they are at rest, the Equation (A.3) becomes

$$
\mu=\frac{\varepsilon}{c^{2}}
$$

\section{Appendix B: Effective Gravity on the Boundary of the Bubble}

Considering a DEMS with an optic wavelength $\lambda=h / p$ produced by the direct interaction of an impinging particle with $\beta=p c / E$ and approaching angle $\theta$, with a antiparticle at rest placed in the lab frame $S$. After the creation of the DEMS occurring during the incoming phase $\mathrm{A}$ in which the source zone localize and emits the energy and the momentum under form of an impulsive electromagnetic signal in radial expansion, begins the successive destroying $\Omega$ phase in which the spatial distance between the two interacting particles grows stretching the value of the effective wavelength of the DEMS at a value $\lambda^{\prime}>\lambda$. Using Eq. (46) and (47), the force felt by an external observer \#1 when it is reached up by the electromagnetic signal emitted by the body $\# 2$ is given by:

$$
\boldsymbol{F}=\mu_{1} \boldsymbol{g}_{2}=-G \frac{\mu_{1} \mu_{2}}{r^{2}} \hat{\boldsymbol{r}}
$$

where $r$ is the effective distance at which observer and particle interact independently by the value of the original wavelength emitted by the DEMS. Assuming for the two bodies an ordinary redshift $z \geq \varsigma_{B G}$, Equation (B.1) is the work done by the electromagnetic forces contrasted by the tension of the vacuum to propagate the original energy of the DEMS. Considering the total strength acting on the observer at the moment in which the wavelength achieves it and the direct interaction starts as 


$$
\boldsymbol{F}=-\operatorname{grad}\left(E_{\Gamma}\right)=-\frac{\mathrm{d} E_{\Gamma}}{\mathrm{d} \lambda} \frac{\mathrm{d} \lambda}{\mathrm{d} r} \hat{\boldsymbol{r}}
$$

the tension of the space-time, depends by wavelength variation, i.e. from the scale variation of the distance of direct interaction

$$
\Psi=-\frac{\mathrm{d} E_{\Gamma}}{\mathrm{d} \lambda} \hat{\boldsymbol{r}}
$$

due to the stretching of the wavelength, hence starting from the definition of the Newtonian force like gradient, is possible to write

$$
\boldsymbol{F}=\Psi \frac{\mathrm{d} \lambda}{\mathrm{d} r}
$$

from which using the Equation (B.1)

$$
\Psi=-G_{0} \frac{\mu_{1} \mu_{2}}{r^{2}} \frac{\mathrm{d} r}{\mathrm{~d} \lambda} \hat{\boldsymbol{r}}
$$

Assuming as distance the Equation (43) and the definition (41), using the redshift definition follows:

$$
\frac{\mathrm{d} r}{\mathrm{~d} \lambda}=\frac{1}{2}\left(\frac{\mathrm{d} \lambda^{\prime}}{\mathrm{d} \lambda}-1+\frac{\lambda_{B G}^{2}}{\lambda^{2}}\right)(1+\beta \cos \phi) .
$$

considering $\lambda^{\prime}=(1+z) \lambda$ and using the Equation (41) the Equation (B.6) becomes

$$
Z=\frac{\mathrm{d} r}{\mathrm{~d} \lambda}=\frac{1}{2}\left(z+\varsigma_{B G}\right)(1+\beta \cos \phi)
$$

that used in the Equation (B.5) rewritten in terms of wavelength i.e. in terms of the initial direct interaction distance gives:

$$
\Psi=-\frac{2\left(z+\varsigma_{B G}\right)}{\left(z-\varsigma_{B G}\right)^{2}(1+\beta \cos \phi)} G_{0} \frac{\mu_{1} \mu_{2}}{\lambda^{2}} \hat{\boldsymbol{r}}=T \boldsymbol{\varphi}
$$

where

$$
\boldsymbol{\varphi}=-G_{0} \frac{\mu_{1} \mu_{2}}{\lambda^{2}} \widehat{\boldsymbol{r}}
$$

is an ideal value of the gravitational force between the two interacting particles in a bubble with wavelength $\lambda$. Using the Equation (47), the Equation (B.8) can be also rewritten in the form

$$
\Psi=Z \boldsymbol{F}
$$

The Equation (B.10) corresponds to the Equation (50). 